\title{
Imposed dynamic irradiation to intensify photocatalytic reactions
}

\author{
Fabian Guba ${ }^{1} \cdot$ Florian Gaulhofer $^{1} \cdot$ Dirk Ziegenbalg ${ }^{1}$
}

Received: 21 January 2021 / Accepted: 3 May 2021 / Published online: 30 June 2021

(C) The Author(s) 2021

\begin{abstract}
Dynamic irradiation is a potent option to influence the interaction between photochemical reactions and mass transport to design high performant and efficient photochemical processes. To systematically investigate the impact of this parameter, the photocatalytic reduction of nitrobenzene was conducted as a test reaction. Dynamic irradiation was realized through provoked secondary flow patterns, multiple spatially distributed light emitting diodes (LEDs) and electrical pulsation of LEDs. A combined experimental and theoretical approach revealed significant potential to enhance photochemical processes. The reaction rate was accelerated by more than $70 \%$ and even more important the photonic efficiency was increased by more than a factor of 4 . This renders imposed dynamic irradiation an innovative and powerful tool to intensify photoreactions on the avenue to large scale sustainable photochemical processes.
\end{abstract}

Keywords Dynamic irradiation · Photocatalysis · Intensification · Reaction engineering · Synchronizing time scales · CFD

\section{Introduction}

Light driven reactions are highly attractive for the development of sustainable chemical processes. Recent developments presented photochemical alternatives for the green production of drugs such as the antimalarial drug artemisinin [1, 2], wastewater and air treatment [3] and organic synthesis [4]. Photons are traceless reagents that cause formation of electronically excited species opening reaction paths that are not accessible through ground state chemistry. By this, reaction sequences can be shortened and energy can be saved. Photoreactions are highly selective and the availability of high power light sources, often in combination with continuous operation, has increased the attractiveness of photochemistry significantly during the last years. [5-11] Photochemical reactions follow the principles of green chemistry to improve sustainability of the overall process by increasing e.g. atom economy, energy efficiency and prevention of chemical waste. [12-17]

While being highly attractive from a birds' perspective, the development of photochemical processes has to include reaction engineering in order to be economical feasible.

Dirk Ziegenbalg

dirk.ziegenbalg@uni-ulm.de

1 Institute of Chemical Engineering, Ulm University, Albert-Einstein-Allee 11, 89081 Ulm, Germany
Compared to thermal reactions, the radiation field has to be considered additionally. This single aspect causes severe implications to the reaction engineering demands. [18] Providing a sufficiently high photon flux to the reaction mixture is the most critical point that must be addressed. [19, 20] This task becomes even more important for photocatalytic reactions involving suspended heterogeneous matter. In addition to the absorption of light, scattering and reflection influence the radiation field in such systems. [21-23] Furthermore, transport processes must be considered and synchronized with the reaction kinetics. Other aspects relevant for conducting heterogeneous photocatalytic reactions include the stability of the suspension and the separation of the photocatalyst from the reaction mixture after the reaction. [24-26]

The interaction of mass transport with photocatalytic reactions is seldom in the focus of research on photocatalytic reactions. The available literature on mass transport effects focuses on the acceleration of the transport of gaseous reactants to enhance the overall reaction rate. [27-32] Given that the intrinsic rate of a photocatalytic process depends on the photon flux, a time dependent change of the incident light intensity represents another option to synchronize the timescales of transport processes and reaction.

Basically, every photocatalytic reaction that is conducted in a stirred vessel or a recycle reactor with suspended catalysts is prone to an unsteady radiation field. This is a direct result of the exponential decay of the light intensity 
by interaction with matter. High intensities are present at the reactor window while the intensity quickly decreases with the distance to the window. Suspended particles move in and out an irradiated section, almost independent of the actual reactor setup. Intense mixing is often found to accelerate the apparent reaction rate. Most frequently, this is attributed to a better suspension quality or a better macroscopic mass transport. [22, 33-36]

Considering this, it is especially surprising that the feasibility of using imposed dynamic irradiation to enhance photocatalytic reactions has hardly been investigated till now. The use of pulsed XeCl-excimer radiation or pulsed laser LEDs are rare examples. [37-41] This is even less comprehensible when considering that photochemical conversions often use solar light that is intrinsically unsteady during the course of the day as well as the year. Consequently, there is a knowledge gap on the impact of an unsteady irradiation on photocatalytic transformations. This knowledge is of high relevance for application as well as the development of photocatalytic active materials.

The photocatalytic synthesis of quinoline is a prime example for the application of the principles of green chemistry. In contrast to conventional multi-step, thermal syntheses such as the Skraup-, Friedländer and DoebnerMiller-syntheses, the photocatalytic synthesis can be conducted as one-pot reaction using UV-A-light $(\lambda=365 \mathrm{~nm})$ at room temperature [42-44]. Contemplating the reaction network, the crucial step is the photocatalytic reduction of nitrobenzene by a photocatalyst, typically titanium dioxide. Through the electron-hole-pairs formed upon irradiation at the catalyst, surface oxidation and reduction reaction are initialized simultaneously. In this consequence, nitrobenzene is reduced by a 6-electron transfer to aniline. The corresponding oxidation product is acetaldehyde, which is generated by oxidation of ethanol. Ethanol is also used as the solvent. In subsequent reaction steps aniline and acetaldehyde undergo acid-catalyzed cyclization reactions to finally reveal the quinoline derivative (see Fig. 1). [45]

With aniline as a member of top 100 utilized chemical synthesis components and quinoline as an important building block for various pharmaceuticals, the photocatalytic quinoline synthesis shows significant potential for a future application in chemical industries [46-48].

For photoreactions in particular, flow chemistry has revealed as a versatile tool for organic synthesis [49-51].
Flow systems offer unique features such as enhanced mass transport and a precise control of residence and irradiation time, by simply adjusting the overall flow rate. Combined with the controlling options of modern LEDemitters, this can be key to a deeper understanding of the underlying molecular and microscopic fundamentals, especially of photocatalytic systems. A fundamental understanding is essential for the development of up-scaling concepts. [52, 53]

In this work, the impact of a dynamic irradiation field on the photocatalytic reduction of nitrobenzene as the first step of the quinoline synthesis is investigated in a flow reactor. A temporal change of irradiation incident on a photocatalyst particle is realized by either the implicit creation of secondary flows, the use of several light sources or pulsed operation of the light source by utilizing rapid prototyping principles. The results show that dynamic irradiation can significantly accelerate the apparent reaction rate of nitrobenzene conversion.

\section{Methods}

\section{Experimental}

For manufacturing the reactor models, a Fused-Deposition Modeling (FDM) 3D-printer (X400 v3, German Reprap $\mathrm{GmbH}$, Germany) was used. Specific components of the printer were modified to address particular requirements for the manufacturing of photoreactors. The original extruder was replaced by a separately acquired model (Titan-extruder, E3D-Online Ltd, UK). Additionally, to increase print quality, a radial fan was installed to cool printed objects. To guarantee adhesion of polypropylene (PP) filament to the printing bed, an adhesive agent (Wolfbite Ultra, AIRWOLF 3D PRINTERS, USA) was applied. A $1.75 \mathrm{~mm}$ diameter, transparent PP filament (Verbatim GmbH, Germany) was used. The top part of the reactor was printed on a MK3S 3D-Printer (Prusa Research a.s., Czech Republic) utilizing $1.75 \mathrm{~mm}$ poly lactic acid (PLA) filament (Prusament, Prusa Research a.s., Czech Republic). An overview of the main printing settings can be found in Table 1 .

The reactor was positioned in a fume hood utilizing milled holdings out of aluminum. As a light source, a 365 nm LED emitter (LZ1-00UV00, LED Engin, Inc.,
Fig. 1 Chemical equation of the photocatalytic reduction of nitrobenzene

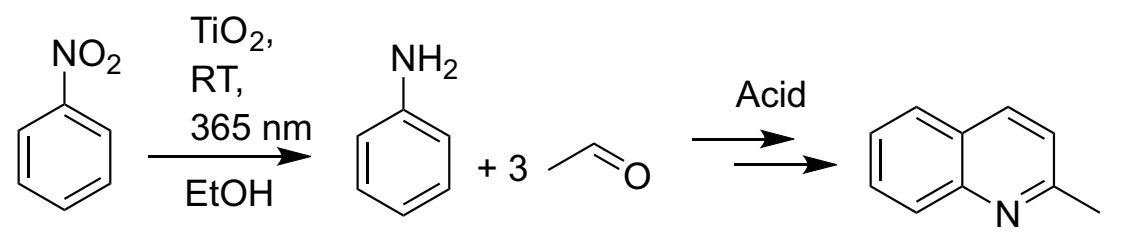


Table 1 Applied 3D-printing settings for both utilized materials (PP and PLA) and printers (X400 and MK3S)

\begin{tabular}{lll}
\hline Parameter & Setting PP (X400) & Setting PLA (MK3S) \\
\hline nozzle diameter & $0.4 \mathrm{~mm}$ & $0.4 \mathrm{~mm}$ \\
extrusion multiplier & 1.03 & 0.95 \\
top layers & 5 & 5 \\
bottom layers & 5 & 4 \\
perimeters & 5 & 5 \\
extrusion width & $0.5 \mathrm{~mm}$ & $0.48 \mathrm{~mm}$ \\
infill percentage & $100 \%$ & $20 \%$ \\
infill pattern & rectilinear & rectilinear \\
nozzle temperature & $230{ }^{\circ} \mathrm{C}$ & $210^{\circ} \mathrm{C}$ \\
bed temperature & $120{ }^{\circ} \mathrm{C}$ & $60{ }^{\circ} \mathrm{C}$ \\
standard printing speed & $25 \mathrm{~mm} \mathrm{~s}{ }^{-1}$ & $50 \mathrm{~mm} \mathrm{~s}^{-1}$ \\
\hline
\end{tabular}

OSRAM, Germany) was utilized equipped with a starshaped aluminum cooler. The cooler was connected to the reactor with elastic fastening bands.

The O-ring gasket of the reactor was constructed from milled parts of aluminum and a $3 \mathrm{~mm}$ thick fluorocarbonbased fluoroelastomer (FKM) O-ring (C. Otto Gehrckens GmbH \& Co. KG, Germany). To cope with the abrasive character of $\mathrm{TiO}_{2}$ a self-constructed peristaltic pump based on a commercial pumping system (CP-86, Gemketechnik $\mathrm{GmbH}$, Germany) using a 3D-printed case with a chemical resistant tubing (Norprene Chemical, Saint-Gobain Performance Plastics Inc., USA) was used. The inner tubing diameter was $4.8 \mathrm{~mm}$ and the outer diameter was $8.0 \mathrm{~mm}$.

For analytics, a deuterium-halogen lightsource (AvaLight-DH-S-BAL, Avantes BV, Netherlands) was used. UV-vis measurements were conducted using fused silica glass cuvettes (inner length: $1 \mathrm{~cm}$ ) in a 3D-printed holder and a connected UV-vis spectrometer (AvaSpec3648, Avantes BV, Netherlands). For solid separation, a centrifuge (CM-70M.07, neoLab Migge GmbH, Germany) was applied.

Chemicals used for nitrobenzene reduction experiments are listed in Table 2. A reaction mixture consisted of nitrobenzene $\left(0.1 \mathrm{~mol} \mathrm{~L}^{-1}\right)$, trifluoromethanesulfonic acid $\left(1 \mathrm{~mol} \mathrm{~L}^{-1}\right)$ and titanium dioxide $\left(0.625 \mathrm{gL}^{-1}\right)$ dissolved in absolute ethanol. Thereby, the raw reaction mixture consisted of only nitrobenzene and trifluoromethanesulfonic acid. $160 \mathrm{~mL}$ of this raw solution was transferred to the reservoir and pumped through the system for $5 \mathrm{~min}$. Subsequently, $100 \mathrm{mg}$ of the catalyst were added. To avoid catalyst agglomerations and precipitations, the suspension was pumped for another 15 min with a flow rate of $\dot{V}=175$ $\mathrm{mL} \min ^{-1}$. Before switching on the LED $(I=0.2 \mathrm{~A})$, a reference sample for an irradiation time of 0 min was taken. After the start of irradiation, samples were taken out of the reservoir every $5 \mathrm{~min}$. To analyze the samples with UV-visspectroscopy, the catalyst was removed by centrifugation at
$7000 \mathrm{~min}^{-1}$ for $30 \mathrm{~min}$. Afterwards samples were measured in a low volume fused-silica-glass cuvette.

Actinometric measurements were conducted with the ferrioxalate actinometer according to literature. [54, 55] Firstly a $0.04 \mathrm{~mol} \mathrm{~L}^{-1}$ ferrioxalate solution was prepared by dissolving iron(III)chloride and potassium oxalate monohydrate in a $0.05 \mathrm{~mol} \mathrm{~L}^{-1}$ aqueous solution of sulfuric acid. $160 \mathrm{~mL}$ of the actinometer solution were transferred to the reservoir of the reactor and pumped through the system. The experiment was started by switching on the LED for 0s, 60s, 105s, 150s, 225s and 300s. After irradiation, a 1 $\mathrm{mL}$ sample was taken and diluted in $24 \mathrm{~mL}$ of a $0.05 \mathrm{~mol}$ $\mathrm{L}^{-1}$ aqueous sulfuric acid solution. $5 \mathrm{~mL}$ of the diluted sample were transferred to $15 \mathrm{~mL}$ of a $0.006 \mathrm{~mol} \mathrm{~L}^{-1} 1,10$ phenanthroline solution in $0.05 \mathrm{~mol} \mathrm{~L}^{-1}$ aqueous sulfuric acid. Subsequently, the samples were left for $1 \mathrm{~h}$ and analyzed utilizing UV-vis-spectroscopy at a wavelength of $510 \mathrm{~nm}$. By the use of a Python script, the photon flux was calculated from the absorbance values. [54, 55]

Placing all devices on the sample port of an $150 \mathrm{~mm}$ integrating sphere (MSP UK150P-REFLTRANS, Mountain Photonics GmbH, Landsberg am Lech, Germany) allowed

Table 2 Chemicals used for nitrobenzene reduction experiments and actinometric measurements.

\begin{tabular}{ll}
\hline Chemical & Supplier \\
\hline nitrobenzene 99.5\% & Acros Organics \\
ethanol $99.98 \%$ & VWR Chemicals \\
2-propanol 98\% & VWR Chemicals \\
$\mathrm{TiO}_{2}$, Aeroxide P25 & Acros Organics \\
iron(III)chloride 98\% & Merck \\
potassium oxalate hydrate $99 \%$ & Alfa Aesar \\
1,10-phenanthroline $99 \%$ & Alfa Aesar \\
sodium acetate $99 \%$ & Sigma Aldrich \\
sulfuric acid $95 \%$ to $97 \%$ & Merck
\end{tabular}


for measuring the transmission and consequently the determination of the extinction coefficient. The integrating sphere was connected to a spectrometer (Ava-SpecULS2048CL-EVO-RS, Avantes BV, Netherlands). First absolute ethanol was pumped through the system and measured as a reference by switching on the LED of the photoreactor. Subsequently, ethanol was removed through the reservoir and a $0.625 \mathrm{gL}^{-1}$ suspension of $\mathrm{TiO}_{2}$ in ethanol was inserted. The suspension was pumped through the system while the light transmission through the reactor was measured.

\section{Computational fluid dynamics}

OpenFOAM v8 with the standard solver pimpleFoam was used for the numerical investigations. [56] The transient application solver was utilized for an incompressible fluid in the laminar flow regime. The presented numerical results are based on the evaluation of the Navier-Stokes equations given in vector form:

$$
\begin{gathered}
\frac{\partial \mathbf{u}}{\partial t}+(\mathbf{u} \cdot \nabla) \mathbf{u}=-\frac{1}{\rho} \nabla p+v \nabla^{2} \mathbf{u} \\
\nabla \cdot \mathbf{u} \quad=0,
\end{gathered}
$$

where $\mathbf{u}$ denotes the velocity field, $\rho$ the constant density of the fluid, $v$ the constant kinematic viscosity of the fluid and $p$ the pressure. The Nabla and Laplace operator are defined as:

$$
\nabla=\left(\frac{\partial}{\partial x}, \frac{\partial}{\partial y}, \frac{\partial}{\partial z}\right), \quad \nabla^{2}=\frac{\partial^{2}}{\partial x^{2}}+\frac{\partial^{2}}{\partial y^{2}}+\frac{\partial^{2}}{\partial z^{2}} .
$$

The spatial fluid regime was defined by three different boundary patches: inlet, outlet and wall. The inlet was initialized for a given flowrate in $y$-direction, the pressure was forced to follow the Neumann boundary condition. The outlet patch set the pressure to be zero and the velocity to follow the Neumann boundary condition. No slip conditions were applied on all wall patches. As fluid ethanol was taken with a kinematic viscosity of $v=1.5122 \cdot 10^{-6}$ $\mathrm{m}^{2} \mathrm{~s}^{-1}$. A first order implicit Euler method was used for time integration together with the adjustable time stepping technique to guarantee a local Courant number (CFL) of CFL $<1$. The general first order setup for space discretisation was used. As numerical solvers "GAMG" was chosen for the pressure and "smoothSolver" for the velocity with absolute tolerances of $1 \cdot 10^{-6}$.

The meshes were generated by the OpenFOAM utility snappyHexMesh. The hexahedral background mesh contained 0.27 million cells. The amount of cells for a solution independent mesh for the different mixing elements varied between 2.68 and 3.07 million. No layer insertion was used. A fine grid near the walls was achieved by surface refinement. All meshes passed the OpenFoam utility checkMesh without error.

\section{Reactor concept and manufacturing}

The impact of dynamic irradiation was investigated in a flow-through reactor with dedicated irradiation sections. For continuous operation, several constraints had to be considered to allow for a thorough analysis of the dynamic irradiation. For continuously operated reactors, a welldefined flow field must be ensured. This requirement was met with laminar flow conditions.

Operating the reactor in a recycle mode through connecting the inlet and outlet to the same reservoir resulted in a differential reactor that gives good access to the reaction kinetics and allows to investigate the effect of different irradiation modes. Furthermore, the flow velocities in sections that are not irradiated should be high to avoid catalyst precipitations. This was realized by using large cross-sectional areas in the irradiation section and reduced cross-sections in all other parts of the setup. For this, a diffuser-nozzle design was chosen for the reactor, involving a central linear unit. The reaction mixture coming from the tubing entered the reactor via an inlet and subsequently the diffuser-unit. To minimize the risk of a developing vortex at the inlet, opening angles of the diffuser were kept as low as possible. However, due to geometrical limitations of the used 3D-printer, the diffuser was made of two sections. The first section was significantly shorter than the second section, resulting also in a larger opening angle. The extended second diffuser section should also work as a buffer, reducing the effect of eventually occurring vortices on the flow conditions in the irradiation zone. The diffuser was connected to the linear part of the reactor. In this section, static mixing elements could be installed and irradiation was conducted. In a standard setup, a $15 \mathrm{~mm}$ by $15 \mathrm{~mm}$ window was installed directly behind the mixing element. The total length of the linear part was $100 \mathrm{~mm}$, allowing various mixing elements to be installed (Fig. 2).

In a modified setup, a second window $(15 \mathrm{~mm}$ by $15 \mathrm{~mm})$ could be positioned at the end of the linear part with a distance of $70 \mathrm{~mm}$ to the end of the first window. In the rear part of the reactor a confuser was installed which is an exact mirror of the inlet diffuser. A CAD-drawing of the assembled reactor is depicted in Fig. 3. In Fig. 4 the reactor placed inside the complete experimental setup is shown. Figure 5 illustrates a P\&I diagram of the setup. The total reactor volume without any installation placed inside was $62.43 \mathrm{~mL}$. At a standard flow rate of $175 \mathrm{~mL}$ $\min ^{-1}$, assuming a plug flow behavior, the residence time in the linear part of the reactor was calculated to be $10.29 \mathrm{~s}$, resulting in a residence time of $1.54 \mathrm{~s}$ inside the irradiation zone below the window.

To increase reproducibility of the measurements, precipitations should be ideally fully avoided, not only in the reactor itself but in the reservoir as well. For this, a cyclone 


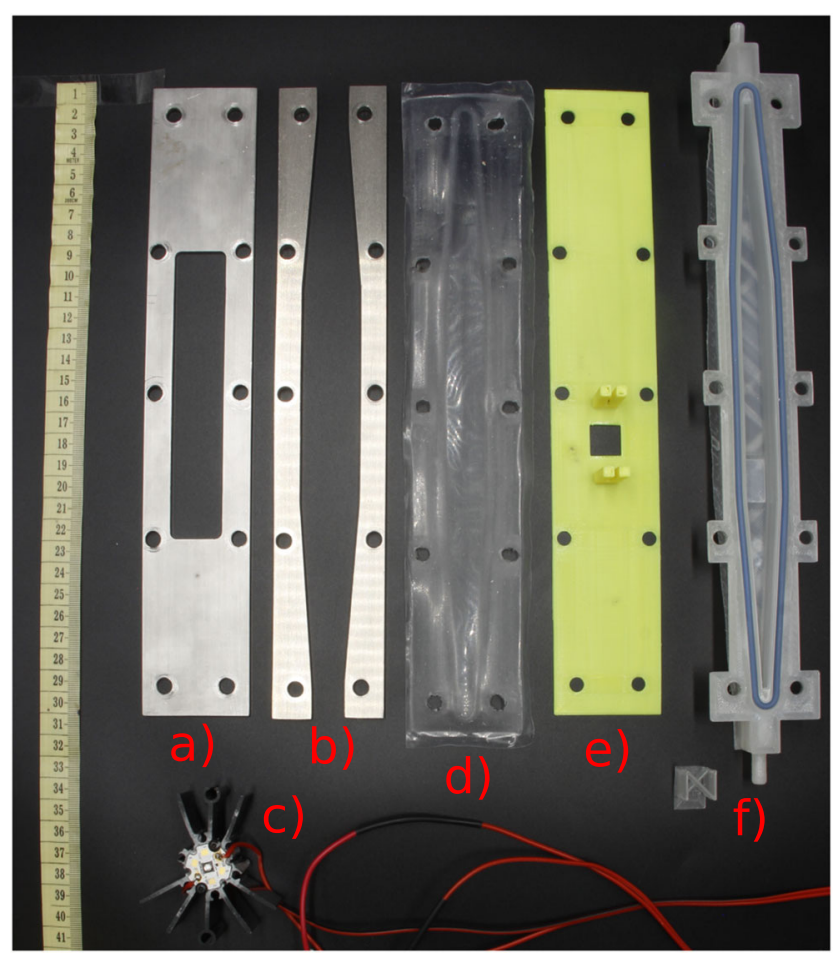

Fig. 2 Fully disassembled reactor. From left to right: Stainless steel top $a$ ) and bottom part $b$ ) of the sealing, utilized 365 nm-LED $c$ ), FEP-film $d$ ), 3D-printed PLA-holding for the LED $e$ ), 3D-printed PP-reactor and a 3D-printed mixing installation $f$ )

shaped reservoir with tangential inflow and an axial outflow was designed and manufactured (see Fig. 6). The induced rotation causes sufficient convection in the reservoir and a stable suspension quality. With this, precipitations could

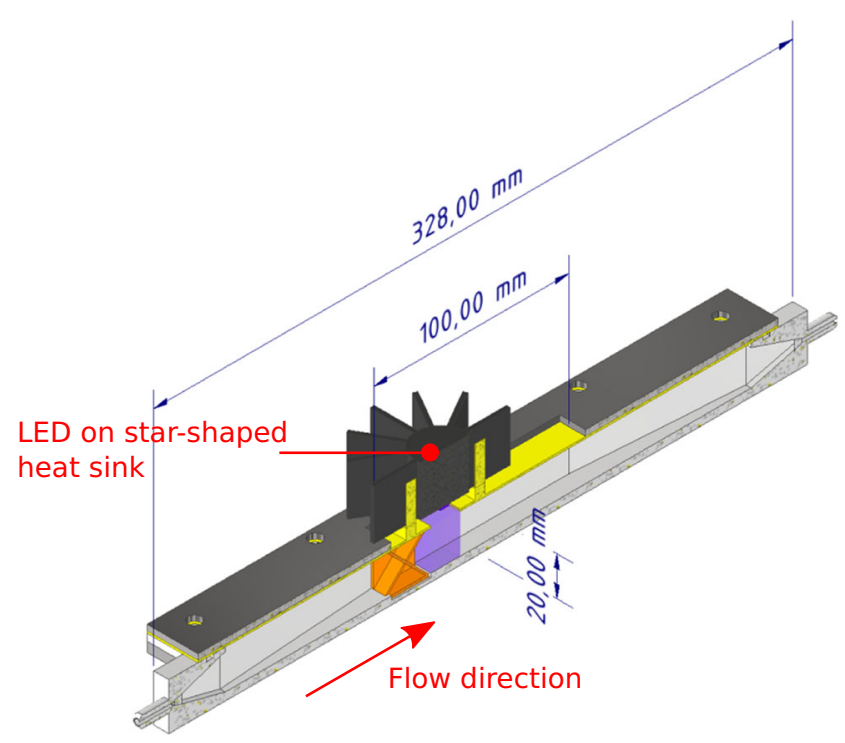

Fig. 3 CAD-drawing of the utilized reactor with inserted "horizontal plates" installation (highlighted in orange). The irradiation area is highlighted in violet

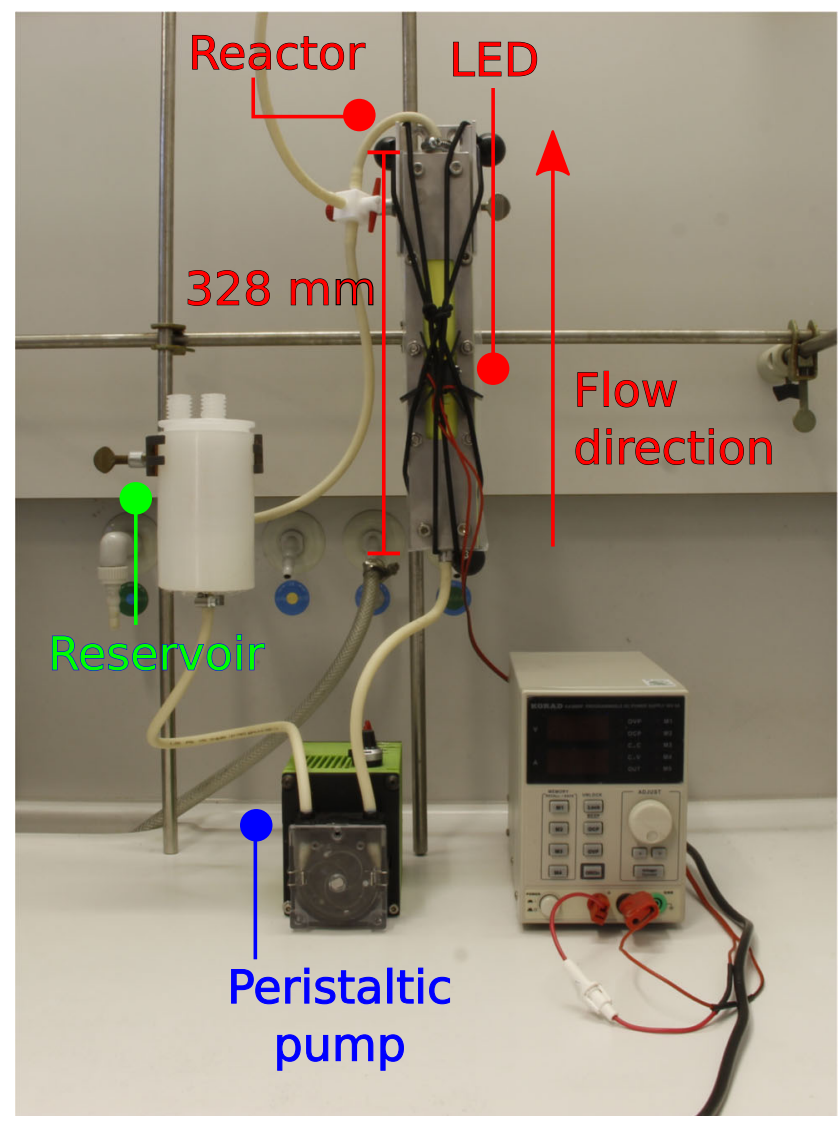

Fig. 4 Experimental setup of the photocatalytic reduction of nitrobenzene. The reaction mixture was pumped from the reservoir in the reactor. Flowing vertically through the reactor, the suspension is guided subsequently from the reactor outlet to the reservoir again. Samples were taken from the reservoir

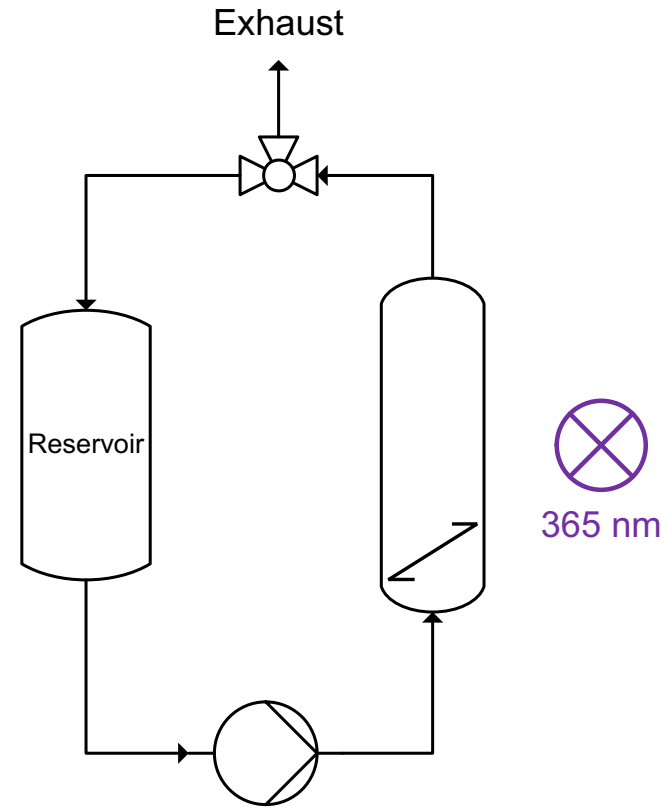

Fig. 5 P\&I-diagram of the experimental setup 


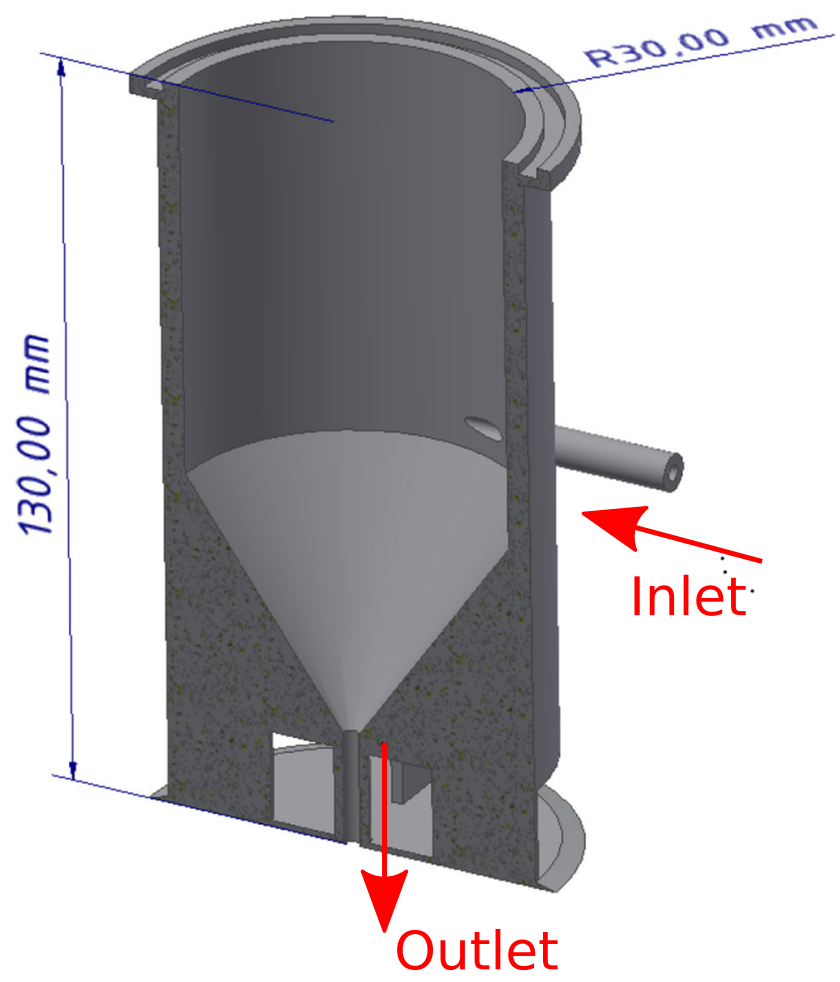

Fig. 6 Cross-section of the new designed and 3D-printed reservoir

be avoided almost completely. Furthermore, no magnetic stirrer is required, simplifying the whole setup. Finally, to shield the reaction mixture from malicious environmental influences, the reservoir could be sealed by a cap sealed with an O-ring.

Fused-depostion-modelling with polypropylene (PP) was used for the manufacturing of the reactor and the reservoir to allow for an easy adaption of the reactor right from the beginning. PP provides a sufficient chemical resistance to e.g nitrobenzene. For reactor models consisting of several parts manufactured out of PP, sealing becomes a crucial point. Compatibility issues with nitrobenzene in the reaction mixture prohibited gluing of the components. Therefore, an O-ring sealing using a chemical resistant fluorinated rubber was installed in the reactor. Additionally, as a side effect, reactor models could be easily reopened to change for example the static mixers and in this way reused.

For photoreactors, a transparent window has to be installed to enable irradiation of the reaction mixture and hence becomes a crucial component. The geometry and composition of the window determines if and how many photons can enter the reactor. For the reactor prototype multiple window types were tested including a fused silica glass, a 3D-printed PP-window and an extruded FEP-foil. Utilizing fused silica glass would have required gluing the glass window into a 3D-printed mounting to reduce the risk of glass breakage. For this again the problem of contaminating the reaction mixture by adhesive agents would arise. A UV-transparent, 3D-printed PP window could not be used due to milli- or micrometer grooves resulting from the printing process that led to random leakages. [57] Alternatively, an extruded FEP-foil was used. The smooth surface of the extruded foil avoided leakages. Finally, to clearly define the irradiation area, an additional PLA-printed cover was installed on top of the FEP-foil that contained holding structures for the LED, allowing for a precise positioning of the light source. With respect to the spatial radiation pattern of the LED and in order to catch a majority of emitted photons, for all reactions the LED was placed in a distance of $6.3 \mathrm{~mm}$, corresponding to a maximum beam angle of $100^{\circ}$.

A photon flux of $q=833 \mathrm{nmol} \mathrm{s}{ }^{-1}$ was measured with actinometry in the reactor when a single LED was operated with an electrical current of $I=0.2 \mathrm{~A}$. The emitted photon flux scaled linearly with the used electrical current.

Dynamic irradiation was realized by three approaches: i) by different hydrodynamic flow patterns, ii) by installation of multiple spatially separated LEDs and iii) by pulsation of the light source. All approaches were meant to provide an unsteady irradiation field to each suspended photocatalyst particle.

The irradiation section is defined by the size of the irradiation window $(15 \mathrm{~mm}$ by $15 \mathrm{~mm})$ and the depth of the channel at this point $(20 \mathrm{~mm})$. A representative particle resides in the irradiation section for a certain time. The period during which a particle is irradiated is defined by the residence time in the section as well as the distance of the particle from the irradiation window. Irradiation stops either when the particle moves outside the window in axial direction of the channel or when the particle moves to a depth in which no light is incident anymore. The impact of these effects might be different since the first case is comparable to switching the light on and off, while the second case goes along with a changing intensity. The simplest way to change the irradiation time is a variation of the flow rate, directly changing the residence time in axial direction. With respect to suspensions stability, this option was not investigated to avoid problems with sedimentation of catalyst particles at lower flow rates. Influencing the flow field is a second option that allows to induce movement of particles in lateral direction as well. [58-64] This was realized with installation of static mixers. To create different flow profiles and with this irradiation patterns, several static mixers were investigated, namely a wall and three cross-shaped mixers.

As first static mixer a wall was investigated that causes a reduction of the cross-sectional area of the channel and consequently an increase of the flow velocity directly behind the wall (referred to as "wall", see Fig. 7a). Additionally, a vortex is expected to form behind the wall. 
Fig. 7 CAD-drawings of the utilized mixing elements that were 3D-printed out of PP. a depicts the 'wall' structure containing a $14 \mathrm{~mm}$ high wall obstacle. $\mathbf{b}$ and $\mathbf{c}$ show single cross-shaped mixers. The structures were combined with a $90^{\circ}$ rotation. $\mathbf{d}$ depicts the 'double mixer' that is a combination of both single cross-shaped mixers

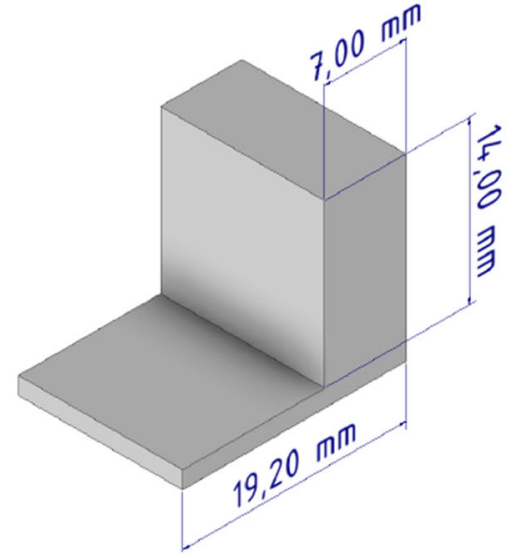

(a)

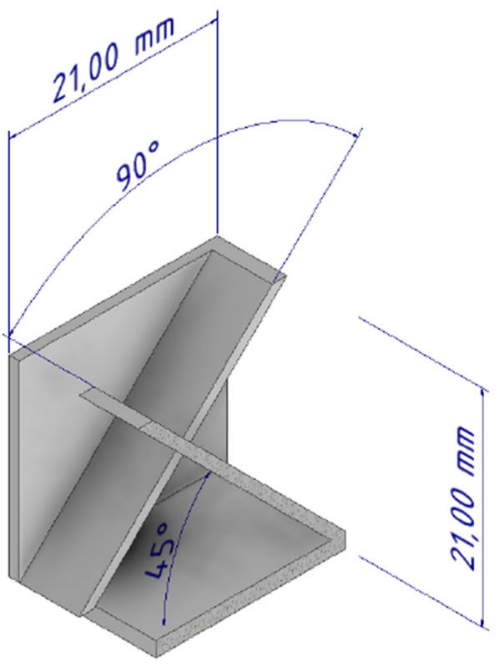

(c)

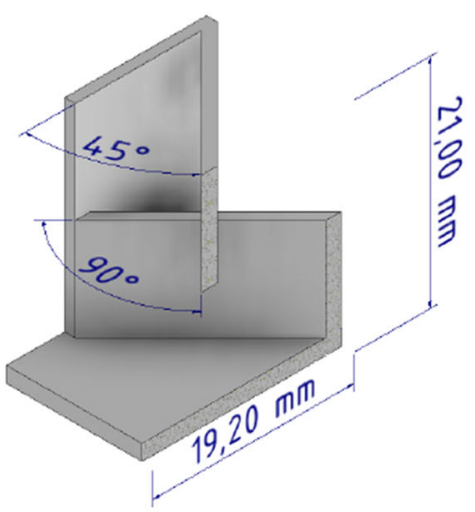

(b)

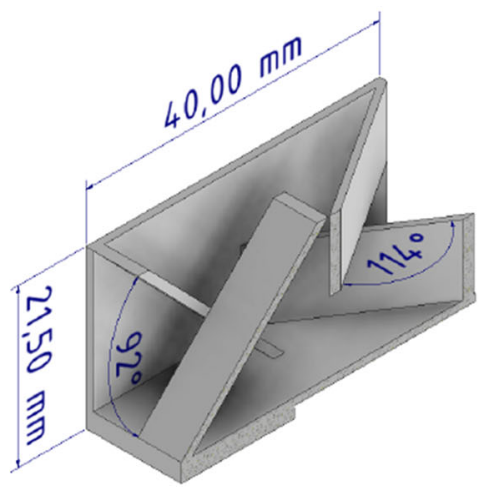

(d)
It is known that cross-shaped mixers, consisting of two crossed plates, are able to generate such helical flow patterns [58, 59, 65-69]. To investigate the impact of such a helical flow, crossed plates were installed in two arrangements, either to induce a vertical or a horizontal movement of the flow (see Fig. 7 band c). This was realized by rotating the crossed plates by $90^{\circ}$. These installations are referred to as "vertical" and "horizontal" plates, indicating the intended main direction of fluid movement. For a third model, both structures were combined and arranged one after the other to generate a more intense rotation (referred to as "double mixer", see Fig. 7d).

The dimensioning of the mixing elements had to respect the limits of the size of the reactor as well as limitations of the 3D-printing process that were given by the nozzle diameter and the minimum printing height. For this, mixing plates had to provide a certain thickness to reveal stable models. Since the mixing elements had full contact to nitrobenzene, the models were manufactured from PP.
Unsteady irradiation was further realized by pulsation of the light source. For this, a $365 \mathrm{~nm}$ UV LED was used.

\section{Transmission measurements}

Suspensions of photocatalytic particles cause absorption and scattering of the incident light. Consequently, the absorption coefficient is not sufficient to describe the interaction of light with matter. A more realistic description can be obtained by determining the extinction coefficient $\beta$ of the used suspension.

Making use of rapid prototyping capabilities, the reactor was adapted such that a second window was installed in the bottom opposite to the first window to enable transmission measurements. Furthermore, the reactor was additionally manufactured with channel heights of $z=$ $10 \mathrm{~mm}$ and $z=5 \mathrm{~mm}$. The results are depicted in Table 3 . No linear correlation was found when considering all 
Table 3 Absorbance measured for different channel heights and calculated extinction coefficient $\beta$ at $365 \mathrm{~nm}$ for a $\mathrm{TiO}_{2}$-suspensions with a loading of $0.625 \mathrm{gL}^{-1}$

\begin{tabular}{lll}
\hline $\mathbf{z} / \mathbf{m m}$ & $\mathrm{A}_{365 \mathrm{~nm}} / \mathbf{1}$ & $\mathrm{A}_{365 \mathrm{~nm}} / \mathbf{m}^{-\mathbf{1}}$ \\
\hline 20 & 2.633 & 232 \\
10 & 2.324 & \\
5 & 1.536 & \\
\hline
\end{tabular}

measured optical depths, indicating that at least the $20 \mathrm{~mm}$ measurement is within analytical limits. Consequently, only optical depths of $z=10 \mathrm{~mm}$ and $z=5 \mathrm{~mm}$ were used to determine the extinction coefficient to $\beta \approx 232 \mathrm{~m}^{-1}$. With this extinction coefficient it can be estimated that around $0.5 \%$ of the light is transmitted after an optical depth of $10 \mathrm{~mm}$.

\section{Results on computational fluid dynamics}

\section{Flow patterns}

Figure 8 depicts 81 representative streamlines for each of the four investigated mixers starting from the outlet backwards. For each mixing element, a 3D view of the streamlines is shown, as well as a side and top view of the reactor. The irradiation window is colored in orange.

The main flow moves in the $y$-direction starting from $-y$ ending at $+y$. A common characteristic flow pattern can be identified for all investigated mixers. After entering the reactor, the majority of streamlines move to either the top or the bottom of the reactor in the first diffusor part and spreads out along the $x$ - and $y$-direction. An unsteady flow pattern emerges because the fluid cannot form a fully developed flow in the diffusor part, causing the streamlines to be present either at the top or bottom. The velocity decreases along the length of the diffusor due to the expansion of the cross-section. The actual position depends on the setup under investigation.

The simulations show that installation of a simple wall leads to the desired effect of directing the fluid towards the irradiation window combined with a reduction of the cross-section (see Fig. 8a). Behind the wall, the fluid velocity decreases and due to kinetic energy loss, the flow destabilizes and forms axial vortices. In general, a chaotic movement of the fluid is observed in the region behind the wall.

The intended formation of a secondary helical flow is found for the vertical plates, the horizontal plates and the double mixer, depicted in Fig. 8b, c, d. For the vertical plates mixer, many streamlines can be found near the irradiation window, similar to the wall mixer. The helical flow starts behind the mixing element and develops after the fluid passed the window. In contrast, the rotation is much more localized behind the mixing element when installing horizontal plates. Helical movement quickly stops behind the irradiation section.

The combined use of horizontal and vertical plates causes an even more intense helical movement of the fluid directly after the mixer as well as an initial guidance of the fluid towards the irradiation window.

\section{Statistical evaluation of streamlines}

Due to significant differences in the flow fields and the requirement to consider the position of the irradiation window, a quantitative comparison of the flow behavior between the different setups is complex and must reflect the impact of the flow field on the irradiation pattern. Such an insight can be gained by statistically evaluating the streamlines, representing the pathway of a fluid element or particle through the reactor projected on the $y z$-plane at $x=$ $0 \mathrm{~m}$. The vertical position of the streamlines (z-direction/ height of the reactor) as well as the time on the streamline can be correlated to the irradiation field that affects the particle. For the evaluation, the frame of reference was defined such that the reactor bottom was positioned at $z=$ $0 \mathrm{~m}$ and the irradiation window at $z=0.02 \mathrm{~m}$.

Around 900 streamlines were started equally spaced at the outlet of all shown devices and calculated backwards to ensure a sufficient number of streamlines behind the mixers. The course of all streamlines that were present behind the mixing elements was evaluated $(y>-0.0115 \mathrm{~m}$ for the double mixer and $y>-0.0295 \mathrm{~m}$ for all others). Figure 9 depicts two representative streamlines of the double mixer. Figure 9a illustrates the helical movement of the fluid and Fig. 9b shows a streamline that does not participate in the helical movement.

To gain a general and quantitative insight into the flow patterns, a statistical evaluation of the properties of maxima in $z$-direction of all streamlines was conducted. Maxima were chosen as they reflect the positions closest to the irradiation window. The extrema were identified by calculating the first derivative of the $z$-position and identification of points were the sign changes. The presence of multiple maxima is a simple indicator for streamlines that show secondary flow. If only one or no maximum is present, the streamline follows a straight course through the reactor, which is similar to the flow pattern when no mixer is installed (compare Fig. 9b). Such streamlines do not contribute to an unsteady irradiation by hydrodynamic means. To reduce the dataset, the following restrictions were applied: i) to consider the results in "Transmission measurements", $z$-coordinates smaller than $10 \mathrm{~mm}$ were excluded, ii) to filter very small variations in height, an 

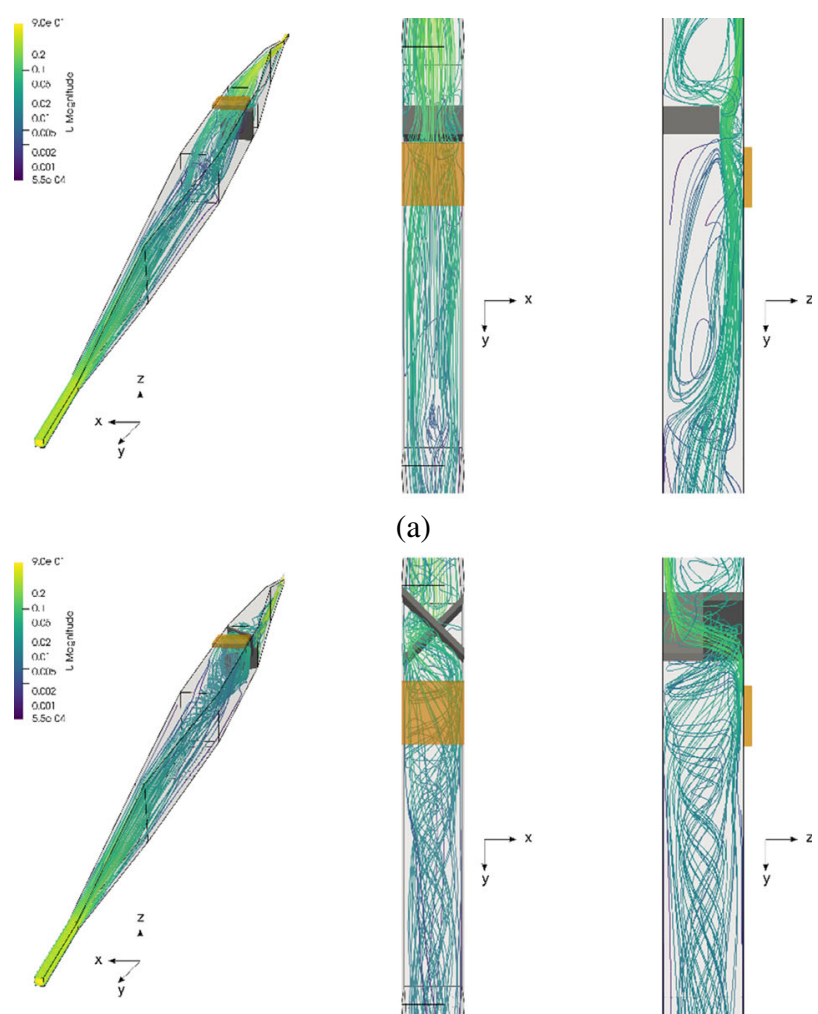

(a)
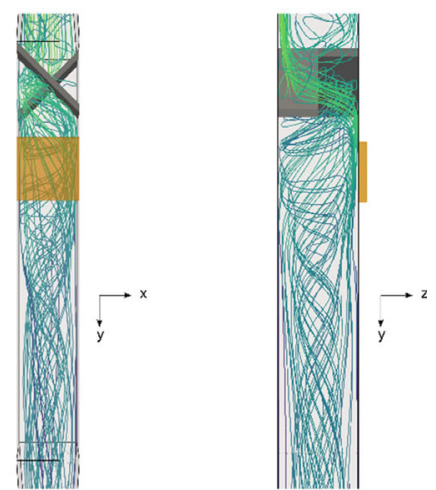

(b)
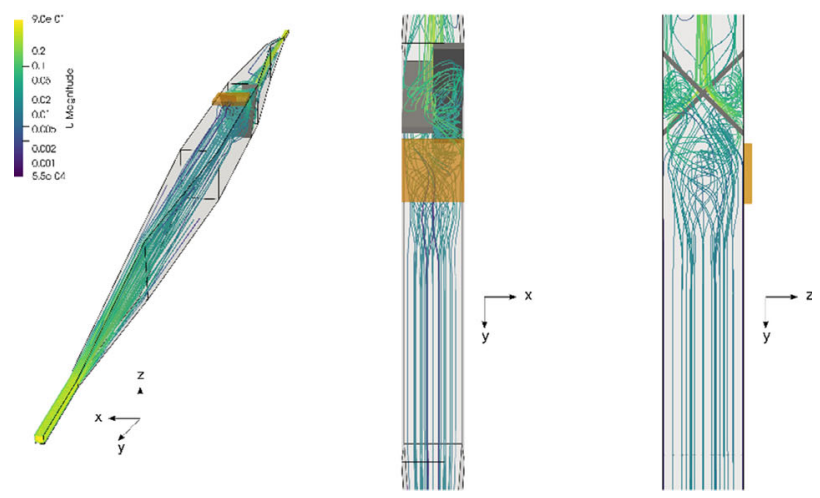

(c)
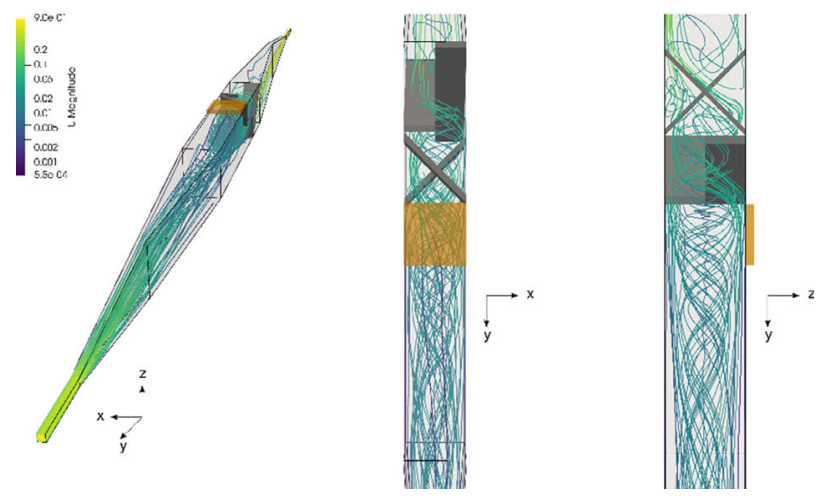

(d)

Fig. 8 Representative streamlines obtained from CFD simulations for a the wall, $\mathbf{b}$ the vertical plates, $\mathbf{c}$ the horizontal plates and $\mathbf{d}$ the double mixer. The fluid enters the reactor from the top and flows in positive $y$-direction. The irradiation window is shown in orange. Streamlines are color coded with the velocity in $y$-direction 
Fig. 9 Representative results of the streamline evaluation for the double mixer, showing the movement in z-direction and the fraction of light transmitted to the streamline. a illustrates a streamline participating and $\mathbf{b}$ a streamline not participating in the helical movement. The lower chart illustrates the transmission on the streamlines

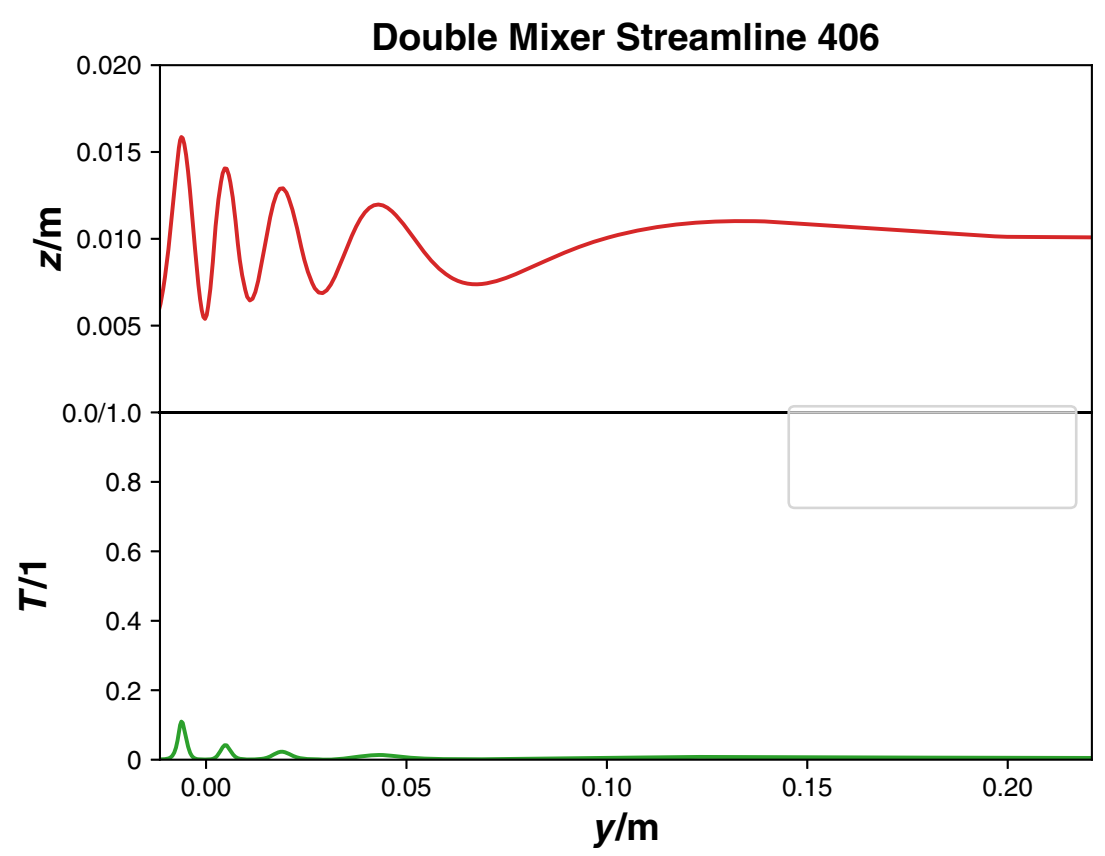

(a)

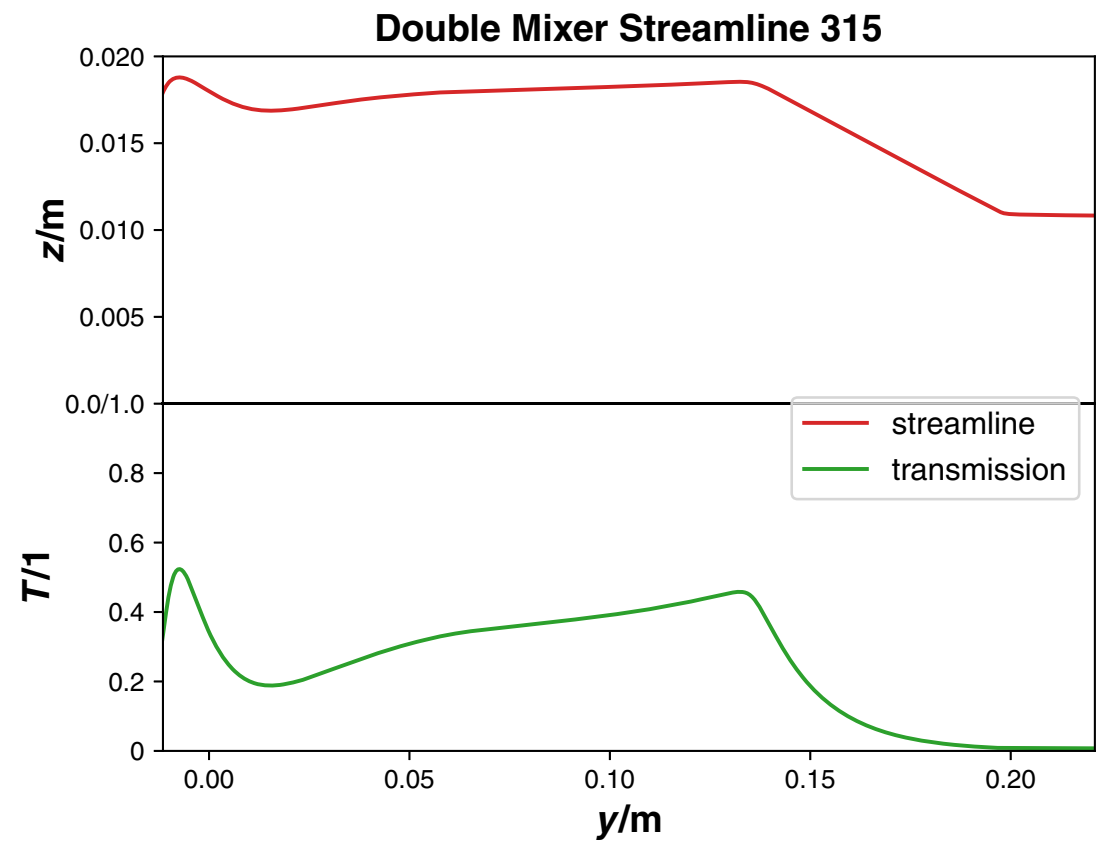

(b) absolute amplitude of the difference in height between two points of $|\Delta z|>0.001 \mathrm{~m}$ was required, iii) evaluation of maxima was restricted to the first $0.1 \mathrm{~m}$ behind the end of the mixer and iv) only streamlines with more than 2 maxima were considered.

The distribution of the mean values of the duration between maxima of each streamline represents a fingerprint of the intensity of helical vertical movement and the irradiation pattern for each device. Figure 10a depicts the results for all setups. In general, a broad distribution of the mean duration is found. As simple metrics for the comparison of the different devices, the mean $\bar{\Pi}$ and median $\tilde{\Pi}$ period of all depicted streamlines were calculated (see Table 4). For the wall mixer, a very broad distribution is found. Due to a frequent occurrence of very short durations between maxima, the median value is much smaller than 
Fig. 10 a: Average time on streamlines between two maxima in $z$-direction (height). b: Average $z$-position (height) of maxima on streamlines. $z=$

$0.02 \mathrm{~m}$ represents the position of the window. c: Average transmission on the streamlines

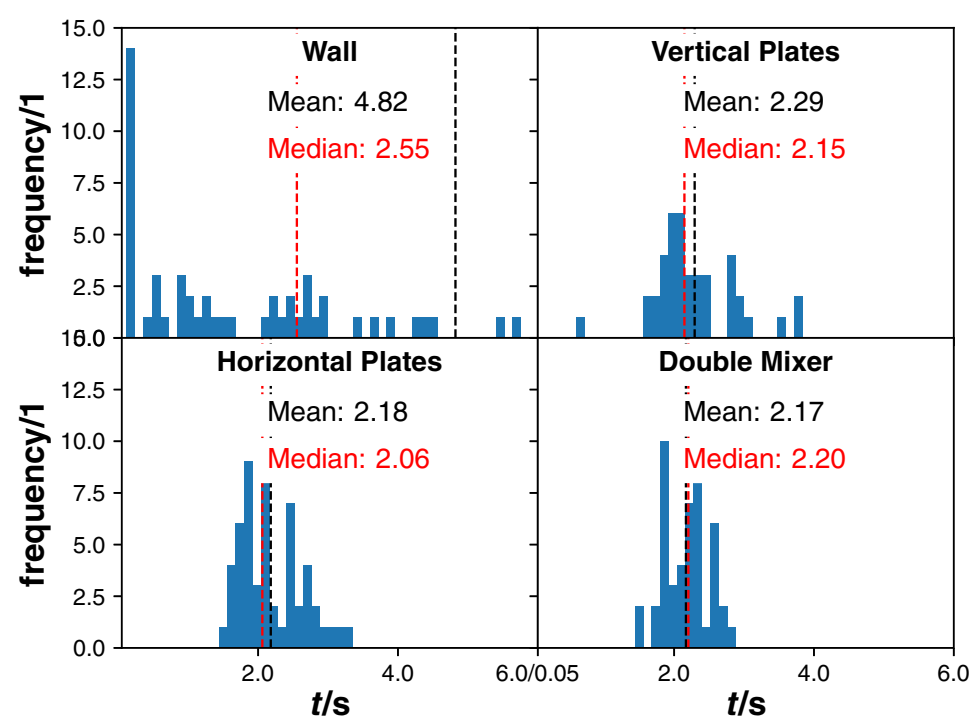

(a)

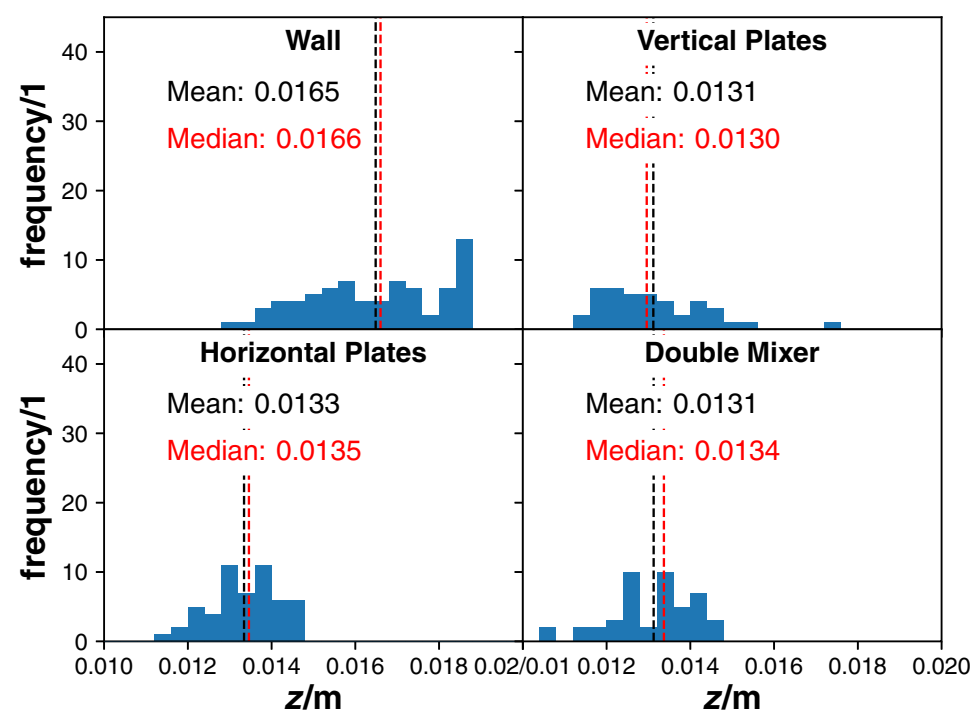

(b)

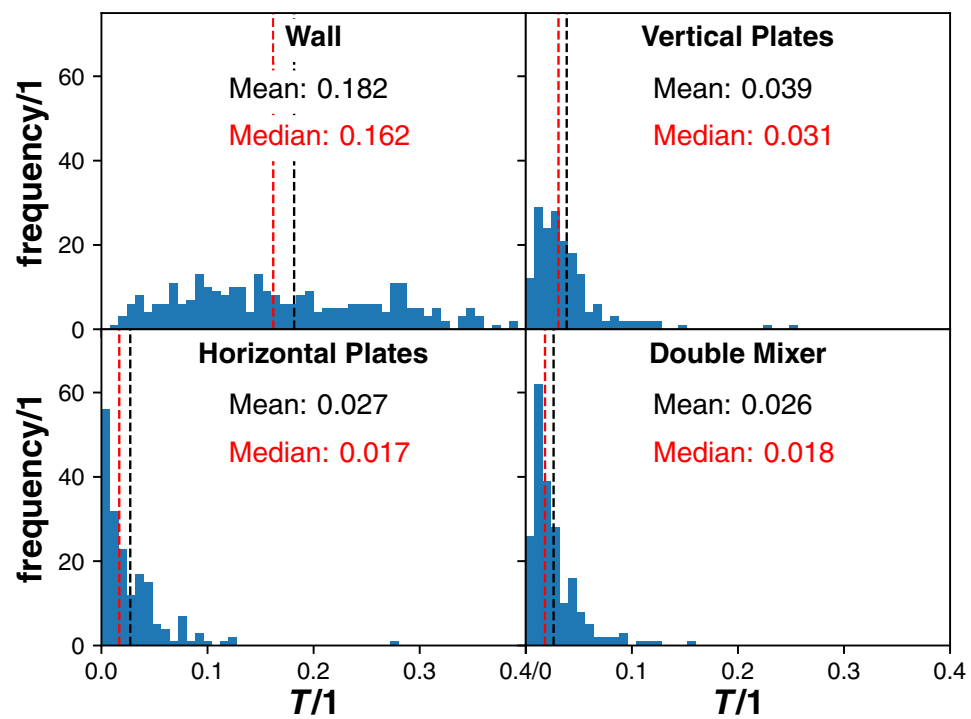

(c) 
Table 4 Metrics for the streamline evaluation for the investigated mixers

\begin{tabular}{lllllrr}
\hline Mixer & $\bar{\Pi} / \mathrm{s}$ & $\tilde{\Pi} / \mathrm{s}$ & $\bar{z} / \mathrm{m}$ & $\tilde{z} / \mathrm{m}$ & $\bar{T} / 1$ & 0.182 \\
\hline Wall & 4.82 & 2.55 & 0.0165 & 0.0166 & 0.162 \\
Vertical Plates & 2.29 & 2.15 & 0.0131 & 0.0130 & 0.039 \\
Horizontal Plates & 2.18 & 2.06 & 0.0133 & 0.0135 & 0.027 & 0.031 \\
Double Mixer & 2.17 & 2.20 & 0.0131 & 0.0134 & 0.026 \\
\hline
\end{tabular}

$\Pi$ represents the period and $z$ the mean z-position of the maxima of the helical flow. $T$ equals the mean transmission on the streamlines. The mean and the median ${ }^{\sim}$ values of all calculated streamlines were calculated for the respective metrics

the mean value. This is a result of the acceleration of the fluid by reducing the cross-sectional area by the wall in combination with extented axial rotations. For the other mixers, the distributions are crowded around the mean value. The longest periods between maxima were found for the wall mixer with $\bar{\Pi}=4.82 \mathrm{~s}$, followed in descending order by the vertical plates, horizontal plates and the double mixer, all showing $\bar{\Pi} \approx 2.2 \mathrm{~s}$.

Beside the period, the position of the maxima in $z$-direction is relevant to elaborate on the impact of the incident photon flux. The mean $z$-position of every identified maxima on every streamline was calculated and is given in Fig. 10a. A broad distribution is found for the wall mixer, while the other devices show a narrower distribution. A comparison of the mean $(\bar{z})$ and median $(\tilde{z})$ values shows the following ascending order: vertical plates mixer, double mixer, horizontal plates mixer and wall mixer (Table 4). The results for the first 3 mixers are almost identical. The mean $z$-position of the wall mixer is with $\bar{z}=0.0165 \mathrm{~m}$ larger than for the other mixers with values around $\bar{z}=0.013 \mathrm{~m}$.

Maxima only represent a snapshot of the irradiation conditions but the "temporal" evaluation of the irradiation field for a moving particle is relevant for the reaction performance as well. To analyze this, the mean transmission $T$ on the course of every streamline behind the static mixer geometry was calculated with the Beer-Lambert law by using the experimentally determined extinction coefficient ("Transmission measurements") and the $z$ position of the streamline. The results are depicted in Fig. 10c. For comparison, the mean $(\bar{T})$ and median $(\tilde{T})$ values of the distribution were calculated. Resulting from the exponential decrease of the light intensity, all setups except for the wall mixer show a pronounced occurrence of low transmission values $(0.026 \leq \bar{T} \leq$ 0.182). The highest mean transmission is found for the wall mixer, followed by the vertical plates mixer, the horizontal plates mixer and the double mixer. For the wall mixer, a very broad distribution of the transmission is found.

\section{Results for nitrobenzene reduction}

The initial reaction rate

$r_{0}=\frac{d n}{d t}$

was calculated for all experiments by linear regression of the results of the first $10 \mathrm{~min}$. To express the apparent efficiency of the reaction, the initial photonic efficiency

$\xi^{0}=\frac{r_{0}}{q_{p, n}^{0}}$

was calculated by normalizing the initial reaction rate with the incident amount based photon flux $q_{n, p}^{0}$. For experiments with pulsed irradiation the time averaged incident photon flux was used.

The conversion of nitrobenzene was calculated with

$X=\frac{n_{0}-n_{30 \mathrm{~min}}}{n_{0}}$,

where $n_{0}$ and $n_{30 \mathrm{~min}}$ is the amount of nitrobenzene at the start of the experiment and after $30 \mathrm{~min}$, respectively.

To simplify discussion, the absolute values of the initial reaction rate will be discussed in the following. Data for all conducted experiments are summarized in Table 5.

The reaction progress is illustrated below for further analysis by drawing the temporal evolution of the amount of nitrobenzene. The presented time span represents the period when the LED emitter was switched on.

In the next sections, the following abbreviations are used: "no mixer" - reactor with no mixer installed; "HP" - horizontal plates mixer; "V-P" - vertical plates mixer, "wall" - wall mixer; "double mixer" - double mixer; "2 LEDs" - reactor irradiated with 2 spatially separated LEDs; "xxx/yyy" - pulsed irradiation with irradiation pulses of xxx ms and pauses of yyy ms; "@ x A" - LEDs were operated with an electrical driving current of $\mathrm{x}$ ampere (A); if no reactor is indicated, the empty reactor was used; combinations of these abbreviations indicate the combination of different techniques to provide a dynamic 
Table 5 Experimental results for the photocatalytic reduction of nitrobenzene

\begin{tabular}{|c|c|c|c|}
\hline Setup & $r_{0} / \mathrm{nmols}^{-1}$ & $\xi 1 \%$ & $X / \%$ \\
\hline no mixer & $11.8 \pm 0.3$ & $1.41 \pm 0.03$ & 81 \\
\hline $\mathrm{H}-\mathrm{P}$ & $11.9 \pm 0.6$ & $1.43 \pm 0.07$ & 84 \\
\hline V-P & $13.0 \pm 0.3$ & $1.56 \pm 0.04$ & 85 \\
\hline wall & $13.3 \pm 0.4$ & $1.60 \pm 0.05$ & 87 \\
\hline double mixer & $13.3 \pm 0.2$ & $1.59 \pm 0.03$ & 89 \\
\hline $\mathrm{H}-\mathrm{P}+15 \mathrm{~mm}$ & 13.1 & 1.57 & 84 \\
\hline $\mathrm{V}-\mathrm{P}+15 \mathrm{~mm}$ & 13.9 & 1.67 & 80 \\
\hline $\mathrm{V}-\mathrm{P}+30 \mathrm{~mm}$ & $14.4 \pm 0.7$ & $1.73 \pm 0.08$ & 83 \\
\hline 2 LEDs@0.1 A & $15.9 \pm 0.3$ & $1.91 \pm 0.04$ & 89 \\
\hline 2 LEDs & $20.0 \pm 0.3$ & $1.20 \pm 0.02$ & 87 \\
\hline $150 / 150$ & $7.8 \pm 1.3$ & $1.87 \pm 0.30$ & 72 \\
\hline $3.9 / 18$ & $9.5 \pm 0.6$ & $6.41 \pm 0.08$ & 71 \\
\hline 3.9/18@1 A & $12.1 \pm 0.5$ & $1.62 \pm 0.07$ & 84 \\
\hline H-P 150/150 & 8.2 & 1.97 & 68 \\
\hline V-P 150/150 & $9.1 \pm 0.5$ & $2.18 \pm 0.11$ & 77 \\
\hline H-P 150/150@0.4 A & 10.6 & 1.27 & 85 \\
\hline V-P 150/150@0.4 A & 12.2 & 1.47 & 83 \\
\hline 2 LEDs 6.25/6.25 & $10.1 \pm 0.8$ & $1.21 \pm 0.10$ & 75 \\
\hline 2 LEDs $3.125 / 3.125$ & 10.4 & 1.25 & 74 \\
\hline
\end{tabular}

If not stated otherwise, LEDs were operated with an electrical current of $I=0.2 \mathrm{~A}$. Results are clustered according to the course of discussion and sorted by $r_{0}$ in ascending order. If not explicitly mentioned, the typical experimental error determined for $r_{0}$ was in the range of $0.05 \mathrm{nmols} \mathrm{s}^{-1}$. This translates to an error of $0.06 \%$ for $\xi$

irradiation. If no electrical driving current is indicated, an electrical current of $0.2 \mathrm{~A}$ was used.

\section{Unsteady irradiation through variation of the flow field}

The results for the reduction of nitrobenzene for the different mixers are shown in Fig. 11. As a base case, the reaction was conducted in a reactor without any mixer installation. The reaction rate in this setup was found to be the slowest of all shown experiments. The reaction rate increases in the order no mixer, horizontal plates mixer, vertical plates mixer, double mixer and wall mixer, while the first two devices and the latter three devices show very similar performance. The initial reaction rate increases from $11.8 \mathrm{nmol} \mathrm{s}^{-1}$ to $13.3 \mathrm{nmol} \mathrm{s}{ }^{-1} \mathrm{~s}$. (Table 5).

Correlating the experimental results with the flow fields (see Fig. 8) reveals that high reaction rates correlate with a more intense convection. A comparison between the horizontal plates and the vertical plates mixer shows that the helicity of the streamlines in the vertical plates mixer is more pronounced than in the horizontal plates mixer.

Since the streamlines depicted in Fig. 8 represent only a share of the streamlines, the streamline analysis given above (see Section 1) complements the evaluation. Interestingly, the period of oscillation is slightly shorter for the horizontal plates mixer than for the vertical plates mixer. This metric correlates the axial to the radial movement of the forming helical flow. Because the superficial velocity was the same in both devices, shorter periods are linked to a more intense radial convection. The slightly faster helical movement does not correlate with the photocatalytic performance. Already from the streamlines depicted in Fig. 8 it is obvious that

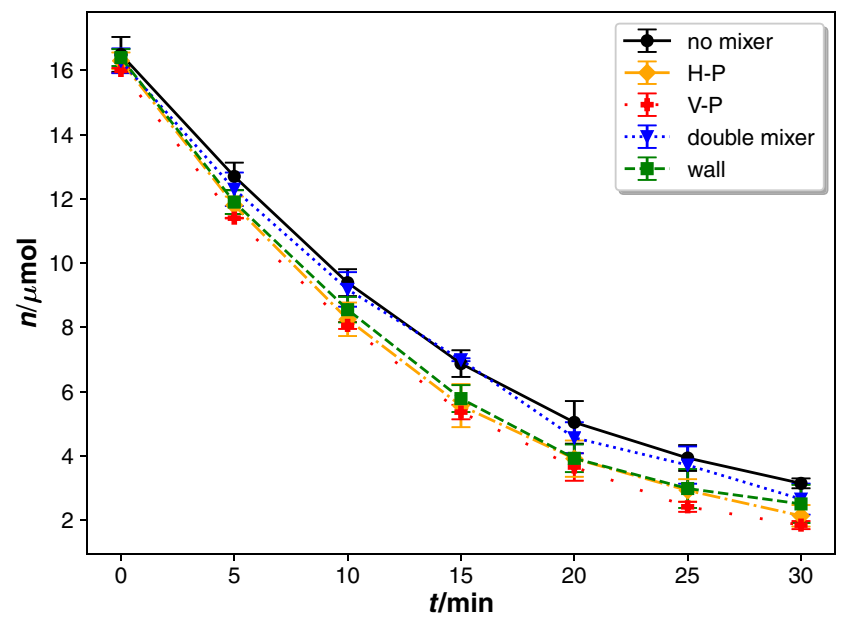

Fig. 11 Amount of nitrobenzene over time of different static mixing elements installed in the flow through reactor. The LEDs were operated with an electrical current of $I=0.2 \mathrm{~A}$ 
the amplitude of the vertical movement is different for both setups but this feature is not reflected in the statistical evaluation as the mean $z$-position of the maxima is similar for both mixer. Hence, from the statistical evaluation of the helical flow, a differentiation between both mixers is not possible.

For the vertical plates mixer, the majority of the streamlines shown in (Fig. 8b) enter the reaction section from the top of the reactor and with this close to the irradiation window, while the streamlines are found to be more homogeneously distributed for the horizontal plates mixer Fig. 8c. Considering the starting rotation of the main flow, particles are faster removed from the window for the vertical mixer as for the horizontal mixer setup. The vertical movement of the streamlines is much more pronounced in the vertical plates mixer. The similar reaction performance of the horizontal plates mixer and the no mixer setup indicates that minor vertical movement of the particles is the common characteristic of these two setups. This leads to the conclusion that vertical movement and with this a shortening of irradiation periods by vertical movement of the particles is important to achieve high reaction rates.

This interpretation is further supported by an evaluation of the mean transmission "incident" on the complete streamlines. A lower mean as well as median transmission is found for the horizontal plates mixer $(\bar{T}=0.027)$ as for the vertical plates mixer $(\bar{T}=0.039)$. It is concluded that convection mainly occurs in regions far away from the light source for the horizontal plates mixer and with this does not support the generation of short irradiation periods.

The initial reaction rate of the double mixer is similar to that of the vertical plates mixer. An even more pronounced helical flow occurs in the double mixer. Since the second element of the double mixer is similar to the vertical plates mixer, the majority of the streamlines are present near the irradiation window and are moving away from the window. Vertical movement starts shortly behind the mixing element and is also present further downstream. The determined period of the helical movement as well as the mean $z$-position of the maxima are similar to the horizontal and vertical plate mixer. It is noteworthy that the mean transmission of the streamlines of the double mixer is similar to the horizontal plates mixer and with this lower as for the vertical plates mixer. The observed higher catalytic performance compared to the horizontal plates mixer gives additional evidence that a fast movement of the particles away from the irradiation window is important.

For the wall mixer, the higher flow velocities due to the reduced cross-sectional area have to be considered additionally. A more intense rotation in axial direction and larger velocities lead to a shortening of the irradiation period compared to the simple pass-by irradiation in a reactor without any mixer installed and consequently to an acceleration of the reaction. The impact is similar as for the vertical plates mixer. Analysis of the streamlines revealed the longest period of oscillation of all setups. This results from the extended axial rotation in combination with low velocities near the bottom of the reactor and close to the wall. The mean height of the maxima is the highest of all mixers. Furthermore, the highest mean transmission was found. Since the type of convection is significantly different from the other mixers, a more detailed analysis is not meaningful.

A comparison of the period of oscillation determined for the different setups with the calculated residence time inside the irradiation section shows that the period of oscillation is always much longer than the residence time. Hence, only a fraction of the movement in $z$-direction occurs in the irradiation zone and a fast vertical movement becomes more important. Considering the required time scale, the results indicate that the irradiation period should be much shorter than the mean residence time below the irradiation window $\tau \approx 1.5 s$.

The intensity of the helical flow behind the mixing elements depends on the distance between the static mixer and the irradiation window. Consequently, this parameter was investigated with reactors that had a shifted position of the irradiation window. The standard position of the window was directly behind the mixing element at a distance of $0 \mathrm{~mm}$. Additionally, the window was installed 15 or $30 \mathrm{~mm}$ behind the mixing element. The results are depicted in Fig. 12 and Table 5.

Shifting the irradiation window for the horizontal and vertical plates mixer leads to an increase of the initial reaction rate. This effect is more pronounced for the horizontal plates mixer, increasing the absolute reaction rate from $11.9 \mathrm{nmol} \mathrm{s}^{-1}$ to $13.1 \mathrm{nmol} \mathrm{s}{ }^{-1}$, comparable to the

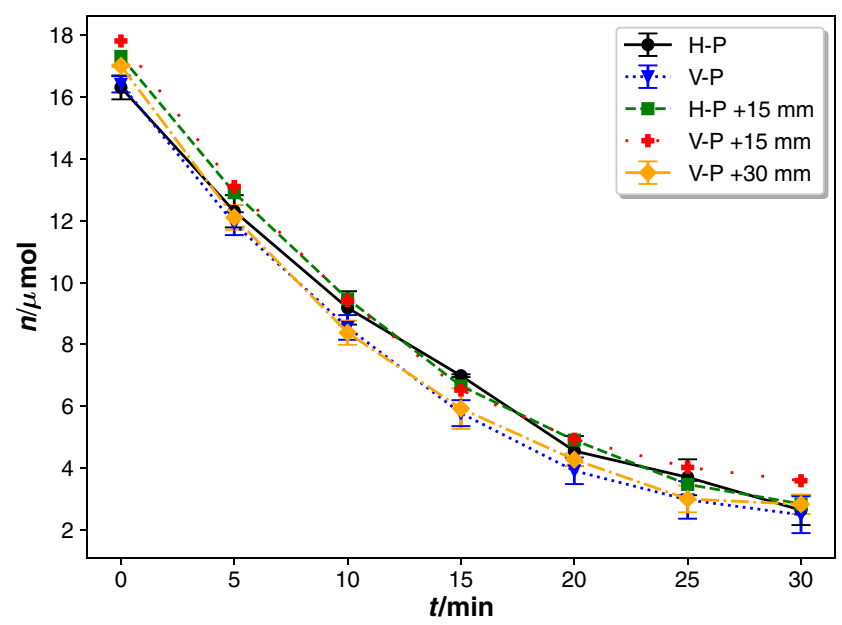

Fig. 12 Amount of nitrobenzene over time for different window positions. The LEDs were operated with an electrical current of $I=$ $0.2 \mathrm{~A}$ 
performance of the vertical plates mixer. Comparison with Fig. 8c indicates that an increased vertical movement is the reason. Repositioning of the irradiation window for the vertical plates mixer increased the reaction rate to $13.9 \mathrm{nmol}$ $\mathrm{s}^{-1}$ and $14.4 \mathrm{nmol} \mathrm{s}{ }^{-1}$ for a distance of $15 \mathrm{~mm}$ or $30 \mathrm{~mm}$, respectively. Since vertical movement is already intense directly behind the mixer, the impact on the reaction rate is minor.

\section{Unsteady irradiation by multiple spatially distributed LEDs}

Installation of two LEDs at different positions is another option to provide pulsed irradiation to the catalyst particles. This was realized by manufacturing another reactor with two irradiation windows, one at the beginning and one at the end of the linear part. To exclude the influence of lateral mixing, no mixing elements were installed.

The catalytic results are depicted in Fig. 13 and Table 5. The reaction rate is significantly increased by the use of two spatially separated LEDs. Initial reaction rates of $r_{0}=15.9 \mathrm{nmol} \mathrm{s}{ }^{-1}$ or even $r_{0}=20 \mathrm{nmol} \mathrm{s}^{-1}$ were found when operating the LEDs with electrical currents of $0.1 \mathrm{~A}$ or $0.2 \mathrm{~A}$, respectively. The reaction progresses almost two times faster than in the empty reactor with a single LED and still faster than in the double mixer. It must be noted, that the incident photon flux is doubled when operating the LEDs with an electrical current of $0.2 \mathrm{~A}$. To consider this, the photonic efficiency $\xi$ must be compared. Operating two LEDs with $0.1 \mathrm{~A}$ or $0.2 \mathrm{~A}$ yields $\xi=1.91 \%$ or $\xi=1.20 \%$, respectively. While the absolute reaction rate is increased by a higher photon flux, the photonic efficiency is decreasing. These values compare to photonic efficiencies of $\xi=1.41 \%$

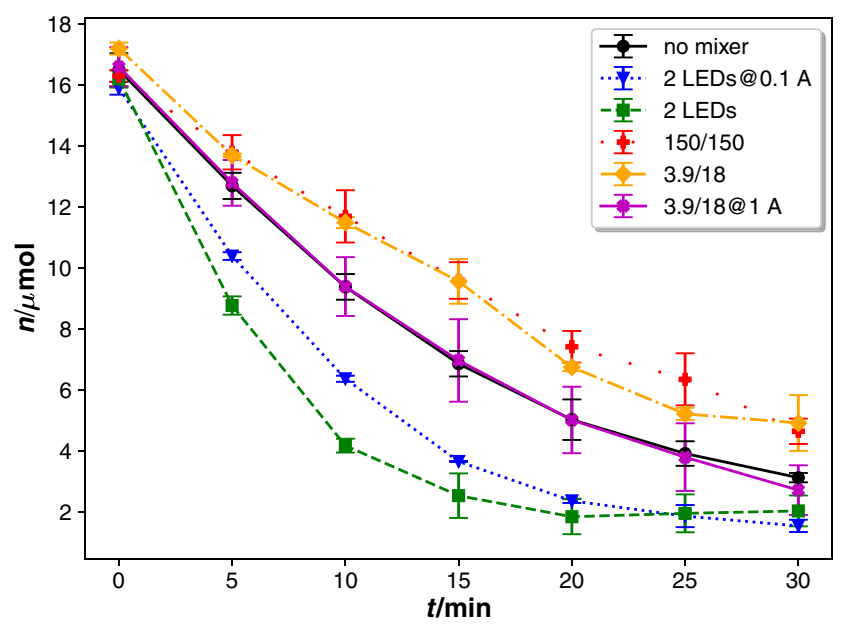

Fig. 13 Amount of nitrobenzene over time for different pulse sequences. If not stated otherwise, LEDs were operated with an electrical current of $I=0.2 \mathrm{~A}$ or $\xi=1.60 \%$ for the reactor without any mixer installed or the wall mixer installed, respectively.

From the geometry and positions of the irradiation windows as well as the flow rate, the duration of irradiation can be estimated to around $1.5 \mathrm{~s}$ together with an irradiation pause of $7.2 \mathrm{~s}$.

\section{Unsteady irradiation by pulsation of LEDs}

Pulsation of the light source is an alternative option to generate an unsteady radiation field and was investigated as well. The results are depicted in Fig. 13. As a base case, irradiation pulses of $150 \mathrm{~ms}$ with similar pause duration were chosen. Following the interpretation above, the duration represents $10 \%$ of the pulse duration estimated for the 2 -LED setup. A reaction rate of around $r_{0}=7.8 \mathrm{nmol} \mathrm{s}^{-1}$ was found, being slower than for the steady irradiation of the empty reactor $r_{0}=11.8 \mathrm{nmol} \mathrm{s}{ }^{-1}$. A slower reaction rate is to be expected since the incident photon flux is halved by the pulsed operation. Notably, the photonic efficiency increases from $\xi=1.41 \%$ to $\xi=1.87 \%$ through the pulsed irradiation. Using a short irradiation pulse of $3.9 \mathrm{~ms}$ together with a long dark period of $18 \mathrm{~ms}$ yields a reaction rate of around $r_{0}=9.5 \mathrm{nmol} \mathrm{s}{ }^{-1}$, even higher than for the experiments with longer and symmetric pulses. The ratio of irradiation and pause duration was chosen similar to the ratio found in the 2-LED setup. Since the time averaged photon flux is significantly lower when using this irradiation sequence, the photonic efficiency significantly increases to $\xi=6.41 \%$. Operating the LEDs with the same pulse sequence but an electrical current of $1 \mathrm{~A}$ provides a five times higher photon flux to the reactor but does not lead to the expected strong increase of the reaction rate. A reaction rate of $r_{0}=12.1 \mathrm{nmol} \mathrm{s}^{-1}$ is found. Consequently, the photonic efficiency drops to $\xi=1.62 \%$.

\section{Combination of methods for unsteady irradiation}

The combination of pulsation of the light source with unsteady irradiation through the flow field was investigated for the vertical and horizontal plates mixer for $150 \mathrm{~ms}$ pulses (see Fig. 14). The reaction rate increases through this combination from $r_{0}=7.8 \mathrm{nmols}^{-1}$ to $r_{0}=8.2 \mathrm{nmols}^{-1}$ and $r_{0}=9.1 \mathrm{nmols}^{-1}$ for the pulsed no mixer configuration, horizontal plates and vertical plates device, respectively. The same order in performance is found as for the steadily irradiated experiments. A further increase of the reaction rate can be realized by increasing the electrical driving current of the LEDs from 0.2A to 0.4A. Compared to experiments with steady irradiation, the absolute reaction rates are in most cases lower. Evaluation of the photonic efficiency reveals high efficiencies of up to $2.18 \%$ for experiments with electrical driving currents of 0.2A. Similar 


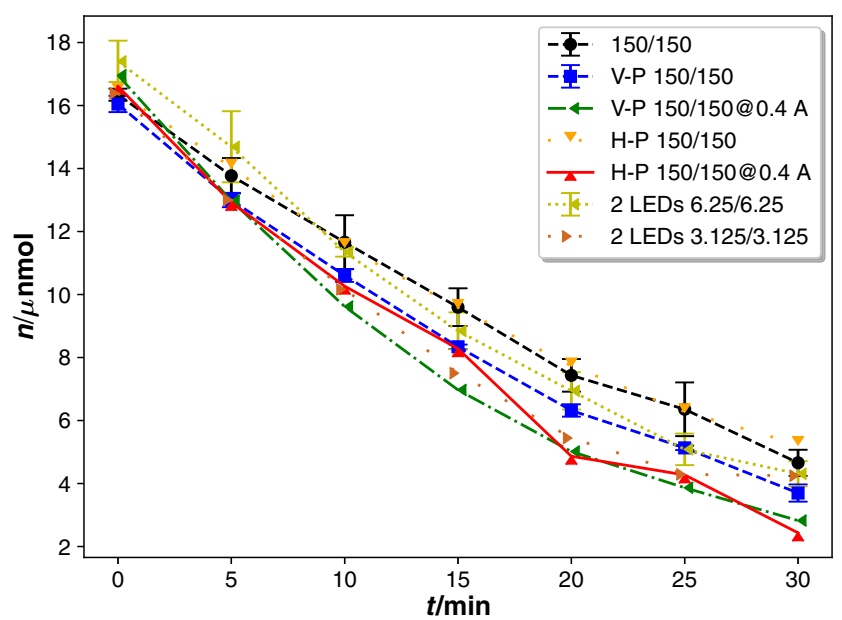

Fig. 14 Amount of nitrobenzene over time for different mixing elements combined with pulse irradiation. If not stated otherwise, LEDs were operated with an electrical current of $I=0.2 \mathrm{~A}$

to all other experiments, the use of high photon fluxes goes along with a decreasing photonic efficiency.

Using two LEDs with pulses of $3.125 \mathrm{~ms}$ or $6.25 \mathrm{~ms}$ and equivalent pauses led to a decrease of the reaction rate. Compared to the steady irradiation case, the reaction rate was almost halved. The photonic efficiency was found to be slightly higher for $3.125 \mathrm{~ms}$ pulse as for the steady case. The opposite was the case for the $6.25 \mathrm{~ms}$ pulse.

\section{General analysis}

To allow for a general analysis of the observed effects, the initial reaction rates and the photonic efficiencies are plotted in Fig. 15. The results are clustered according to the above discussion and sorted in ascending order within these clusters.

An intense convection generally increases the reaction rate, independent if an unsteady irradiation is provided through switching LEDs or not (see Fig. 15a). The discussion above clearly showed that movement of catalyst particles away from the light source is required. This has to be ensured through suited flow patterns. When utilizing provoked secondary flows, the position of this section has to be aligned with the hydrodynamics.

The use of spatially distributed LEDs can further increase the initial reaction rate. Operating LEDs in a pulsed mode generally decreases the reaction rate since a lower time averaged photon flux is provided to the reactor. Combining unsteady irradiation by different flow patterns and pulsed light sources can be used synergistically to accelerate the reaction. Short irradiation together with longer dark periods was found to be beneficial. In general, operating LEDs with larger electrical driving currents leads to higher reaction rates.

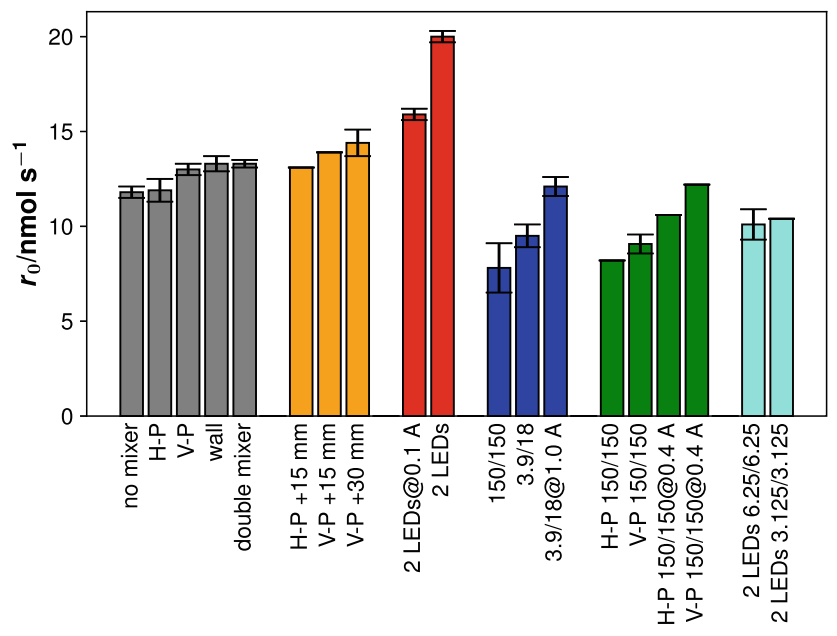

(a)

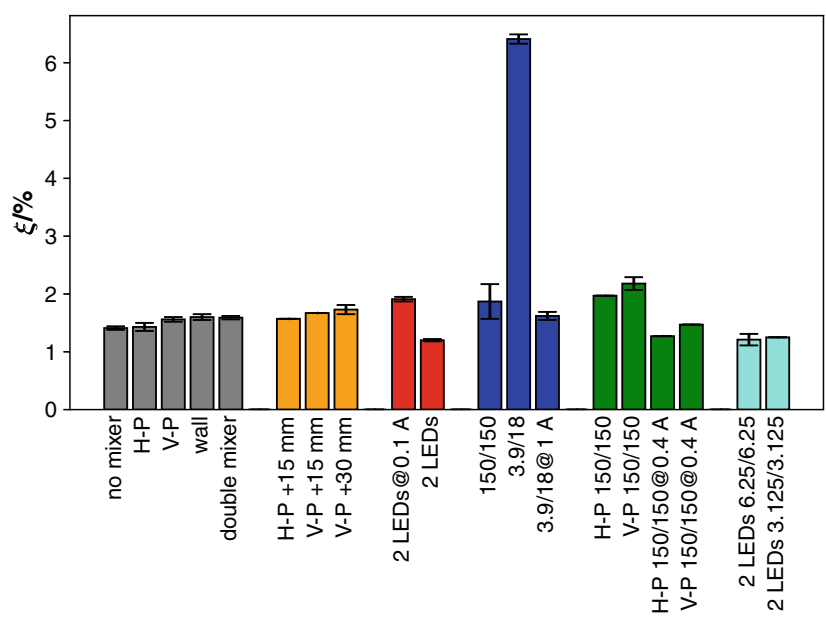

(b)

Fig. 15 a Initial rate constants $r_{0}$ and $\mathbf{b}$ photonic efficiency $\xi$ of the investigated setups results are clustered according to the discussion in the text and sorted in ascending order within the clusters. If not stated otherwise, LEDs were operated with an electrical current of $I=0.2 \mathrm{~A}$

Focusing on the photonic efficiencies it becomes evident that providing an unsteady irradiation field to the catalyst particles leads to an increase of the efficiency (see Fig. 15b). Controlling the hydrodynamics is an effective option to increase the photonic efficiency. Since the combination of pulsed operation of the LEDs and provoked secondary flows and operation of the LEDs with very short irradiation pulses yielded even higher efficiencies, it can be concluded that very short irradiation pulses in the range of several milliseconds are required to gain the highest photonic efficiencies. The results indicate that hydrodynamic removal of particles from the irradiation zone is not sufficiently fast for a high performance.

For a thorough interpretation of the observed effects, it has to be considered that photocatalytic processes involve 
the formation of electrons and holes upon excitation. These charge carriers recombine on a very short time scale if a reaction is not possible. It is known that the quantum yield under periodic irradiation increases to a value equal to that observed for the same (lower) average incident photon flux under continuous irradiation. [70, 71] In the complex reaction network of the nitrobenzene reduction, it is further possible that mass transport effects play a role. If charge separation is induced by excitation but the product blocks the access to the catalyst surface, recombination will occur, leading to a lower photonic efficiency. When increasing the incident photon flux, the rate of charge separation and with this the density of electrons and holes increases. Two counter-acting effects occur for this situation. The probability of inducing a reaction increases due to the larger charge density but with this the probability of charge recombination as well. If the removal of the product from the surface is slow, an additional rate limiting step occurs and consequently, the photonic efficiency decreases.

Realization of unsteady irradiation by hydrodynamic means and the use of multiple light sources are concepts that are very promising for the development of high performance photoreactors. This is nicely illustrated for the setup with 2 LEDs operating with an electrical driving current of $I=0.1 \mathrm{~A}$. For the same incident photon flux, this setup shows an about $30 \%$ higher initial reaction rate and with this also a higher photonic efficiency compared to the no mixer setup. While realization of unsteady irradiation by pulsing the light sources leads to an overall reduction of the incident photon flux and with this capacity of the reactor, the aforementioned concepts do not show this limitation. Instead, the temporal irradiation profile for individual particles can be tuned independent of the time-dependent light emission of the light source. This opens new degrees of freedom for the design of photoreactors.

\section{Conclusion and outlook}

While being a white spot in the field of photochemical reaction engineering, unsteady irradiation of photocatalysts proved to be a very potent approach to accelerate the overall reaction rate of photoreactions. The presented combined theoretical and experimental approach shows for the first time that hydrodynamic manipulations of the flow field can be used to generate an imposed unsteady radiation. A short irradiation period was found to be crucial for the investigated photocatalytic reduction of nitrobenzene. Optimized irradiation conditions lead to an increase of the reaction rate by $70 \%$ related to the reference case of steady irradiation with no mixing element installed and even more important to an enhancement of the photonic efficiency by a factor larger than 4. For the reduction of nitrobenzene, the required irradiation period should be rather short, namely in the lower milliseconds range. Since, the highest reaction rates were not associated with the highest photonic efficiency, further systematic investigations are required to achieve the necessary synchronization on a macro scale.

It is plausible that the need for synchronizing the irradiation profile with the microscopic rate determining step is the most important point for all heterogeneously catalyzed photoreactions. The relevant step strongly depends on the reaction under investigation, including electronic process, electron transfer, adsorption, desorption or diffusion. This renders dynamic irradiation, independent of the way it is realized, a powerful tool to exploit the huge potential of photochemical reactions. A thorough understanding of the interaction of all involved processes with photoreactions is required to identify optimal irradiation patterns as well as reactor designs and scale-up concepts.

Acknowledgements This work was supported through the German Ministry of Economic Affairs and Energy (BMWi), based on a resolution of the German Parliament within the AiF/IGF project QuinoLight, grant no. $18904 \mathrm{~N} / 3$ and N/5 and the AiF/ZIM project MISCOP, grant no. ZF4654701ZG8.

The authors thank Marvin Backes and Dennis Stucke for their help with experimental work and Abhinav Nagarajan for his help with simulations.

Funding Open Access funding enabled and organized by Projekt DEAL.

\section{Declarations}

Conflict of interest The authors declare that they have no conflict of interest.

Open Access This article is licensed under a Creative Commons Attribution 4.0 International License, which permits use, sharing, adaptation, distribution and reproduction in any medium or format, as long as you give appropriate credit to the original author(s) and the source, provide a link to the Creative Commons licence, and indicate if changes were made. The images or other third party material in this article are included in the article's Creative Commons licence, unless indicated otherwise in a credit line to the material. If material is not included in the article's Creative Commons licence and your intended use is not permitted by statutory regulation or exceeds the permitted use, you will need to obtain permission directly from the copyright holder. To view a copy of this licence, visit http://creativecommons. org/licenses/by/4.0/.

\section{References}

1. Triemer S, Gilmore K, Vu GT, Seeberger PH, Seidel-Morgenstern A (2018) Literally green chemical synthesis of artemisinin from plant extracts. Angew Chem Int Ed 57(19):5525-5528. https://doi.org/10.1002/anie.201801424

2. Amara Z, Bellamy JFB, Horvath R, Miller SJ, Beeby A, Burgard A, Rossen K, Poliakoff M, George MW (2015) Applying green 
chemistry to the photochemical route to artemisinin. Nat Chem 7(6):489-495. https://doi.org/10.1038/nchem.2261

3. Hernández-Alonso MD, García-Rodríguez S, Suárez S, Portela R, Sánchez B, Coronado JM (2011) Highly selective one-dimensional TiO2-based nanostructures for air treatment applications. Appl Catal B Environ 110:251-259. https://doi.org/10.1016/j.apcatb.2011.09.009

4. Pfoertner K-H, Oppenländer $T$ (2010) Photochemistry. In: Ullmann's encyclopedia of industrial chemistry. Wiley, Chichester

5. Sugimoto A, Sumino Y, Takagi M, Fukuyama T, Ryu I (2006) The barton reaction using a microreactor and black light. continuous-flow synthesis of a key steroid intermediate for an endothelin receptor antagonist. Tetrahedron Lett 47(35):61976200. https://doi.org/10.1016/j.tetlet.2006.06.153

6. Knowles JP, Elliott LD, Booker-Milburn KI (2012) Flow photochemistry: Old light through new windows. Beilstein $\mathrm{J}$ Organic Chem 8:2025-2052. https://doi.org/10.3762/bjoc.8.229

7. Beatty JW, Douglas JJ, Miller R, McAtee RC, Cole KP, Stephenson CRJ (2016) Photochemical perfluoroalkylation with pyridine n-oxides: Mechanistic insights and performance on a kilogram scale. Chem 1(3):456-472. https://doi.org/10.1016/j.chempr. 2016.08.002

8. Mizuno K, Nishiyama Y, Ogaki T, Terao K, Ikeda H, Kakiuchi K (2016) Utilization of microflow reactors to carry out synthetically useful organic photochemical reactions. J Photochem Photobiol C: Photochem Rev 29:107-147. https://doi.org/10.1016/j.jphotochemrev.2016.10.002

9. Junkers T, Wenn B (2016) Continuous photoflow synthesis of precision polymers. React Chem Eng 1(1):60-64. https://doi.org/10.1039/C5RE00042D

10. Sender M, Ziegenbalg D (2017) Light sources for photochemical processes - estimation of technological potentials. Chem Ing Tech 89(9):1159-1173. https://doi.org/10.1002/cite.201600191

11. Shvydkiv O, Jähnisch K, Steinfeldt N, Yavorskyy A, Oelgemöller M (2018) Visible-light photooxygenation of $\alpha$-terpinene in a falling film microreactor. Catal Today 308:102-118. https://doi.org/10.1016/j.cattod.2017.11.009

12. Hoffmann N (2008) Photochemical reactions as key steps in organic synthesis. Chem Rev 108(3):1052-1103. https://doi.org/10.1021/cr0680336

13. Albini A, Fagnoni M (2008) The greenest reagent in organic synthesis: Light: Green chemical reactions. NATO Science for Peace and Security Series C: Environmental Security, pp 173-189. https://doi.org/10.1007/978-1-4020-8457-7_8

14. Protti S, Dondi D, Fagnoni M, Albini A (2009) Assessing photochemistry as a green synthetic method. carbon-carbon bond forming reactions. Green Chem 11(2):239-249. https://doi.org/10.1039/b810594d

15. Noyori R (2010) Insight: Green chemistry: the key to our future. Tetrahedron 66(5):1028. https://doi.org/10.1016/j.tet.2009.11.021

16. Anastas P, Eghbali N (2010) Green chemistry: Principles and practice. Chem Soc Rev 39(1):301-312. https://doi.org/10.1039/ b918763b

17. Jackson WR, Campi EM, Hearn MTW (2017) Green chemistry: Challenges and opportunities, pp 943-1007

18. Guba F, Tastan U, Gugeler K, Buntrock M, Rommel T, Ziegenbalg D (2019) Rapid prototyping for photochemical reaction engineering. Chem Ing Tech 91(1-2):17-29. https://doi.org/10.1002/cite.201800035

19. Meir G, Leblebici ME, Fransen S, Kuhn S, Gerven TV (2020) Principles of co-axial illumination for photochemical reactions: Part 1. model development. J Adv Manuf Process 2(2). https://doi.org/10.1002/amp2.10044

20. Bonfield HE, Knauber T, Lévesque F, Moschetta EG, Susanne F, Edwards LJ (2020) Photons as a 21 st century reagent. Nat
Commun 11(1):804. https://doi.org/10.1038/s41467-019-13988-4, https://www.nature.com/articles/s41467-019-13988-4

21. Spadoni G, Bandini E, Santarelli F (1978) Scattering effects in photosensitized reactions. Chem Eng Sci 33(4):517-524. https://doi.org/10.1016/0009-2509(78)80012-8

22. Cassano AE, Martin CA, Brandi RJ, Alfano OM (1995) Photoreactor analysis and design: Fundamentals and applications. Indust Eng Chem Res 34(7):2155-2201. https://doi.org/10.1021/ ie00046a001

23. Megatif L, Dillert R, Bahnemann DW (2020) Determination of the quantum yield of a heterogeneous photocatalytic reaction employing a black body photoreactor. Catal Today 355:698-703. https://doi.org/10.1016/j.cattod.2019.06.008

24. Alfano OM, Romero RL, Cassano AE (1986) Radiation field modelling in photoreactors-II. heterogeneous media. Chem Eng Sci 41(5):1137-1153. https://doi.org/10.1016/0009-2509(86) 87087-7

25. Schneider J, Bahnemann D, Ye J, Puma GL, Dionysiou DD (eds) (2016) Photocatalysis. Royal Society of Chemistry, London

26. Bloh JZ, Marschall R (2017) Heterogeneous photoredox catalysis: Reactions, materials, and reaction engineering. Eur J Org Chem 2017(15):2085-2094. https://doi.org/10.1002/ejoc.201601591

27. Herrmann J-M (1999) Heterogeneous photocatalysis: fundamentals and applications to the removal of various types of aqueous pollutants. Catal Today 53(1):115-129. https://doi.org/10.1016/s0920-5861(99)00107-8

28. Alfano OM, Bahnemann D, Cassano AE, Dillert R, Goslich $R$ (2000) Photocatalysis in water environments using artificial and solar light. Catal Today 58(2-3):199-230. https://doi.org/10.1016/s0920-5861(00)00252-2

29. Dijkstra MFJ, Michorius A, Buwalda H, Panneman HJ, Winkelman JGM, Beenackers AACM (2001) Comparison of the efficiency of immobilized and suspended systems in photocatalytic degradation. Catal Today 66(2-4):487-494. https://doi.org/10.1016/s0920-5861(01)00257-7

30. Kako T, Nakajima A, Watanabe T, Hashimoto K (2005) Comparison of photocatalytic properties of a batch reactor with those of a flow reactor in a nearly controlled mass transport region. Res Chem Intermed 31(4-6):371-378. https://doi.org/10.1163/1568567053956572

31. Kopetzki D, Levesque F, Seeberger PH (2013) A continuousflow process for the synthesis of artemisinin. Chem-a Eur J 19(17):5450-5456. https://doi.org/10.1002/chem.201204558

32. Loponov KN, Lopes J, Barlog M, Astrova EV, Malkov AV, Lapkin AA (2014) Optimization of a scalable photochemical reactor for reactions with singlet oxygen. Organ Process Res Dev 18(11):1443-1454. https://doi.org/10.1021/op500181z

33. Parrino F, Bellardita M, García-López EI, Marcì G, Loddo V, Palmisano L (2018) Heterogeneous photocatalysis for selective formation of high-value-added molecules: Some chemical and engineering aspects. ACS Catal 8(12):11191-11225. https://doi.org/10.1021/acscatal.8b03093

34. Shidpour R (2015) A power-law relationship between characteristics of light source and quantum yield in photocatalytic systems. J Phys Chem C 119(39):22425-22431. https://doi.org/10.1021/acs.jpcc.5b02318

35. Camera-Roda G, Loddo V, Palmisano L, Parrino F (2017) Guidelines for the assessment of the rate law of slurry photocatalytic reactions. Catal Today 281:221-230. https://doi.org/10.1016/j. cattod.2016.06.050

36. Rehm TH (2020) Flow photochemistry as a tool in organic synthesis. Chem Eur J 26(71):16952-16974. https://doi.org/10.1002/ chem. 202000381

37. Silvares AFM, do Nascimento CAO, Oliveros E, Bossmann SH, Braun AM (2007) Pulsed $\mathrm{XeCl}$ excimer radiation 
for optimizing the polydispersity of methyl methacrylate pre-polymers. Indust Eng Chem Res 46(23):7436-7447. https://doi.org/10.1021/ie070129i

38. Ouchi A, Sakai H, Oishi T, Kaneda M, Suzuki T, Saruwatari A, Obata $T$ (2008) Photochemical reduction of flavone with nabh4 in batch and micro-channel reactors using excimer lasers. J Photoch Photobio A 199(2-3):261-266. https://doi.org/10.1016/j.jphotochem.2008.05.024

39. Ouchi A, Hyugano T, Kaneda M, Suzuki T, Liu C (2014) Twostep laser photolysis of flavone and $\mathrm{NaBH} 4$ at organic-aqueous liquid interface using a microchannel reactor: A method to avoid secondary thermal side reactions. J Flow Chem 4(4):190-194. https://doi.org/10.1556/jfc-d-14-00025

40. Taştan Ü, Ziegenbalg D (2021) Photochlorination of toluene - the thin line between intensification and selectivity. part 1: intensification and effect of operation conditions. Reaction Chemistry \& Engineering. https://doi.org/10.1039/d0re00263a

41. Taştan Ü, Seeber P, Kupfer S, Ziegenbalg D (2021) Photochlorination of toluene - the thin line between intensification and selectivity. part 2: selectivity. Reaction Chemistry \& Engineering. https://doi.org/10.1039/d0re00366b

42. Hakki A, Dillert R, Bahnemann D (2009) Photocatalytic conversion of nitroaromatic compounds in the presence of $\mathrm{TiO} 2$. Catal Today 144(1-2):154-159. https://doi.org/10.1016/j.cattod. 2009.01.029

43. Hakki A, Dillert R, Bahnemann DW (2013) Factors affecting the selectivity of the photocatalytic conversion of nitroaromatic compounds over $\mathrm{TiO} 2$ to valuable nitrogen-containing organic compounds. Phys Chem Chem Phys 15(8):2992. https://doi.org/10.1039/c2cp44153e

44. Selvam K, Swaminathan M (2011) One-pot photocatalytic synthesis of quinaldines from nitroarenes with au loaded TiO nanoparticles. Catal Commun 12(6):389-393. https://doi.org/10.1016/j.catcom.2010.11.004

45. Patzsch J, Berg B, Bloh JZ (2019) Kinetics and optimization of the photocatalytic reduction of nitrobenzene. Front Chem 7. https://doi.org/10.3389/fchem.2019.00289

46. Afzal O, Kumar S, Haider MR, Ali MR, Kumar R, Jaggi M, Bawa S (2015) A review on anticancer potential of bioactive heterocycle quinoline. Eur J Med Chem 97:871-910. https://doi.org/10.1016/j.ejmech.2014.07.044

47. Marella A, Tanwar OP, Saha R, Ali MR, Srivastava S, Akhter M, Shaquiquzzaman M, Alam MM (2013) Quinoline: A versatile heterocyclic. Saudi Pharmaceut J 21(1):1-12. https://doi.org/10.1016/j.jsps.2012.03.002

48. Kouznetsov V, Mendez L, Gomez C (2005) Recent progress in the synthesis of quinolines. Curr Org Chem 9(2):141-161. https://doi.org/10.2174/1385272053369196

49. Rehm TH (2020) Flow photochemistry as a tool in organic synthesis. Chem (Weinheim Bergstrasse German) 26(71):1695216974. https://doi.org/10.1002/chem.202000381

50. Thomson CG, Lee A-L, Vilela F (2020) Heterogeneous photocatalysis in flow chemical reactors. Beilstein J Organ Chem 16:1495-1549. https://doi.org/10.3762/bjoc.16.125

51. Jang S, Jung B-J, Kim M-J, Lee W, Kim D-P (2019) Reactionvolume dependent chemistry of highly selective photocatalytic reduction of nitrobenzene. React Chem Eng 4(10):1752-1756. https://doi.org/10.1039/c9re00251k

52. Sezen-Edmonds M, Tabora JE, Cohen BM, Zaretsky S, Simmons EM, Sherwood TC, Ramirez A (2020) Predicting performance of photochemical transformations for scaling up in different platforms by combining high-throughput experimentation with computational modeling. Organic Process Res Dev 24(10):21282138. https://doi.org/10.1021/acs.oprd.0c00182

53. Corcoran EB, McMullen JP, Lévesque F, Wismer MK, Naber JR (2020) Photon equivalents as a parameter for scaling photoredox reactions in flow: Translation of photocatalytic c-n cross-coupling from lab scale to multikilogram scale. Angewandte Chemie (Int Engl) 59(29):11964-11968. https://doi.org/10.1002/anie.201915412

54. Wriedt B, Ziegenbalg D (2020) Common pitfalls in chemical actinometry. J Flow Chem 10(1):295-306. https://doi.org/10.1007/s41981-019-00072-7

55. Wriedt B, Kowalczyk D, Ziegenbalg D (2018) Experimental determination of photon fluxes in multilayer capillary photoreactors. ChemPhotoChem 2(10):913-921. https://doi.org/10.1002/cptc.201800106

56. OpenFOAM Foundation (2020) Openfoam, userguide version 8, openfoam foundation. https://openfoam.org

57. Sender M, Ziegenbalg D (2021) Radiometric measurement techniques for in-depth characterization of photoreactors - part 2: 3 dimensional and integral radiometry. under review

58. Pahl MH, Muschelknautz E (1980) Statische mischer und ihre anwendung. Chem Ing Tech 52(4):285-291. https://doi.org/10.1002/cite.330520402

59. Zalc JM, Szalai ES, Muzzio FJ, Jaffer S (2002) Characterization of flow and mixing in an SMX static mixer. AIChE J 48(3):427436. https://doi.org/10.1002/aic.690480303

60. Stroock AD (2002) Chaotic mixer for microchannels. Science 295(5555):647-651. https://doi.org/10.1126/science.1066238

61. Thakur RK, Vial C, Nigam KDP, Nauman EB, Djelveh G (2003) Static mixers in the process industries-a review. Chem Eng Res Des 81(7):787-826. https://doi.org/10.1205/026387603322302968

62. Ghanem A, Lemenand T, Valle DD, Peerhossaini H (2014) Static mixers: Mechanisms, applications, and characterization methods - a review. Chem Eng Res Des 92(2):205-228. https://doi.org/10.1016/j.cherd.2013.07.013

63. Hermann $\mathrm{P}$, Timmermann J, Hoffmann M, Schlüter $M$, Hofmann C, Löb P, Ziegenbalg D (2017) Optimization of a split and recombine micromixer by improved exploitation of secondary flows. Chem Eng J 334:1996-2003. https://doi.org/10.1016/j.cej.2017.11.131

64. Lin C-M, Chang Y-W (2020) Optimization designation of static mixer geometry considering mixing effect. Microsyst Technol. https://doi.org/10.1007/s00542-020-04962-y

65. Myers KJ, Bakker A, Ryan D (1997) Avoid agitation by selecting static mixers. Chem Eng Prog 93(6):28-38

66. Regner M, Östergren K, Trägårdh C (2005) An improved numerical method of calculating the striation thinning in static mixers. Comput Chem Eng 30(2):376-380. https://doi.org/10.1016/j. compchemeng.2005.09.006

67. Liu S, Hrymak AN, Wood PE (2005) Design modifications to SMX static mixer for improving mixing. AIChE J 52(1):150-157. https://doi.org/10.1002/aic.10608

68. Singh MK, Anderson PD, Meijer HEH (2009) Understanding and optimizing the SMX static mixer. Macromol Rapid Commun 30(4-5):362-376. https://doi.org/10.1002/marc.200800710

69. Meijer HEH, Singh MK, Anderson PD (2012) On the performance of static mixers: A quantitative comparison. Prog Polym Sci 37(10): 1333-1349. https://doi.org/10.1016/j.progpolymsci.2011.12.004

70. Cornu CJG, Colussi AJ, Hoffmann MR (2001) Quantum yields of the photocatalytic oxidation of formate in aqueous $\mathrm{TiO} 2$ suspensions under continuous and periodic illumination. J Phys Chem B 105(7):1351-1354. https://doi.org/10.1021/jp003204a

71. Prozzi M, Sordello F, Barletta S, Zangirolami M, Pellegrino F, Prevot AB, Maurino V (2020) Assessing a photocatalytic activity index for $\mathrm{TiO} 2$ colloids by controlled periodic illumination. ACS Catal 10(16):9612-9623. https://doi.org/10.1021/acscatal.0c02518

Publisher's note Springer Nature remains neutral with regard to jurisdictional claims in published maps and institutional affiliations. 